Supplementary Online Material

1 Ash data set

2 Dating errors

3 Statistical Analyses

4 Stress calculations

5 References 


\section{Ash Data Set}

In the attached table we list tephra layers used in our statistical analysis. The tephra layers along the "Ring of Fire" (ROF) are taken from the preliminary reports of DSDP, ODP and IODP cruises. Tephra layers within the last $490 \mathrm{ka}$ in offshore Central America are taken from Kutterolf et al. (2008) and are complemented by data available from DSDP Leg 67 (Sites 499 and 496) for which erosional effects are less abundant. The record is extended by older, dated layers from multiple ODP reports (Aubouin et al., 1982a, 1982b, 1982c; Bowles et al., 1973; Kimura et al., 1997). Ages estimated for tephras obtained on cruises M66, M54, SO173 and ODP Leg 170 (Clift et al., 2005), and their respective correlations to land tephras (Kutterolf et al., 2008), are given in Table DR1.

In compiling the tephra layer data, we first accounted for layers (L), then pod-layers (P), and then observable layers of dispersed ash (disperse). If ash horizons appeared at a same age in two neighbouring sites, we selected the one with a stronger representation. Dispersed ash directly above ash layers is interpreted as being reworked from this layer. Dispersed ash layers from adjacent cores at similar depth (within several $\mathrm{dm}$ ) are interpreted to be from one event unless they differ compositionally in glass chemistry. Ash layers of similar ages in neighbouring cores are also treated as a single event unless there are compositional or structural (thicknesses, grain sizes) differences and the distance of the locations is $<50 \mathrm{~km}$.

At Nankai sites, we used data from Site 1173 from the incoming plate (since it showed less evidence of erosion) and complemented this with the record from Site 1174, the deepest site in the trench (Shipboard, 2000). Relatively well-preserved tephras from the interval covered at Site 1173 were preferred due to signs of major turbiditic reworking at Site 1174. On the other hand, prominent layers at Site 1174 were preferred over their dispersed pendants at Site 1173. Furthermore, in the older legs of Alaska, the Aleutian Arc and Basin, and Kamchatka (Creager and Scholl, 1973a, 1973b, 1973c; Kulm and von Huene, 1973a, 1973b), no proper smear slides were created and therefore diatom/foraminifer rich sands/silts are not evaluated regarding ash abundance that are often coupled with them (Creager and Scholl, 1973a, 1973b, 1973c).

For offshore North New Zealand, we used data for the last $30 \mathrm{kyr}$ from an offshore study (Lowe et al., 2008) that samples in their distribution fans a region that includes the ODP drill site 1124 (Shipboard, 1998); the latter includes tephras down to $1 \mathrm{Ma}$. At Site 1124, there is a gap in the tephra record between ca. 200ka to $450 \mathrm{ka}$ due to washout during coring. For the Tonga Arc we used and merged the tephra records of two nearby ODP Sites 836 and 837 (Parson et al., 1992). Data from the Philippines Sites 767 to 770 (Rangin et al., 1990) have been merged to obtain a nearly continuous tephra record for the Philippines. 
For tephras ages listed in the table below, we use published age models when combined foraminifera ages (Bio) and palaeomagnetic ages (Pmag) are available - for example, at younger DSDP, ODP and IODP drill sites. In contrast, we typically use biogenic ages alone in the older DSDP sites, where paleomagnetic studies have not been possible or have large errors (see discussion below).

Additionally, ages obtained through correlation with land tephras (Corr), or from sedimentation rates (sed) between well-dated marine tephras, have been used to establish sedimentation rates at the CAVA drill sites. Varying sedimentation rates with depth at one drill site are constrained (e.g. site 496: $100 \mathrm{~cm} / \mathrm{kyr}$ for the first 3 cores and, subsequently, $14 \mathrm{~cm} / \mathrm{kyr}$ ) such that the total age equals the sum of the age in the first section characterized by high sedimentation rate and the age derived from the deeper part of the core characterized by smaller sedimentation rate. Previous correlations between tephras in CAVA gravity cores (Kutterolf et al. 2008) to DSDP/ODP sites are listed in the column "site/correlative site" within the table. In the column "age model method", the method used to determine the tephra ages is listed first, followed by the dating method for the correlative tephra/horizon. ${ }^{14} \mathrm{C}$ ages are from charcoal and soils, ${ }^{40} \mathrm{Ar}{ }^{39} \mathrm{Ar}$ dates are from kalifeldspars. The second row in this column lists the errors for the correlative tephras/horizons. The names of field tephras and relevant publications are listed in the right column. 
Table DR1: Data table of tephra layers used for this study

\begin{tabular}{|c|c|c|c|c|c|c|c|c|c|c|c|c|}
\hline Region & cruise & Leg & $\begin{array}{c}\text { Site/ } \\
\text { Correlative } \\
\text { Site }\end{array}$ & $\begin{array}{l}\text { Sed. } \\
\text { rate } \\
(\mathbf{c m} / \mathbf{k a})\end{array}$ & core & section & $\begin{array}{l}\text { depth } \\
(\mathrm{cm})\end{array}$ & $\begin{array}{l}\text { depth bsf } \\
(\mathrm{cm})\end{array}$ & $\begin{array}{l}\text { calculated } \\
\text { Age from } \\
\text { sed. rates }\end{array}$ & $\begin{array}{l}L=\text { layer, } \\
P=\text { pods, } \\
L(P)= \\
\text { pods layer }\end{array}$ & $\begin{array}{l}\text { Age model } \\
\text { method (Bio, } \\
\text { pmag, sed, Corr) }\end{array}$ & $\begin{array}{l}\text { correlated to } \\
\text { onshore tephra } \\
\text { (only CAVA and } \\
\text { New Zealand) }\end{array}$ \\
\hline \multirow[t]{18}{*}{ Peru } & DSDP & 202 & $1237 \mathrm{~B} / \mathrm{C}$ & 2.5 & 1 & 1 & 5 & 5 & 2.0 & disperse & Bio/pmag & \\
\hline & DSDP & 202 & $1237 \mathrm{C}$ & 2.5 & 1 & 1 & 21 & 21 & 8.4 & disperse & Bio/pmag & \\
\hline & DSDP & 202 & $1237 \mathrm{~B}$ & 2.5 & 1 & 3 & 120 & 420 & 168.0 & $\mathrm{~L}$ & Bio/pmag & \\
\hline & DSDP & 202 & $1237 \mathrm{~B}$ & 2.5 & 1 & 4 & 44 & 494 & 197.6 & $\mathrm{~L}$ & Bio/pmag & \\
\hline & DSDP & 202 & $1237 \mathrm{~A}$ & 2.5 & 1 & 3 & 125 & 525 & 210.0 & $\mathrm{~L}$ & Bio/pmag & \\
\hline & DSDP & 202 & $1237 \mathrm{C}$ & 2.5 & 1 & 5 & 20 & 620 & 248.0 & $\mathrm{~L}$ & Bio/pmag & \\
\hline & DSDP & 202 & $1237 \mathrm{~A} / \mathrm{D}$ & 2.5 & 1 & 3 & 90 & 660 & 264.0 & $\mathrm{~L}$ & Bio/pmag & \\
\hline & DSDP & 202 & $1237 \mathrm{D}$ & 2.5 & 1 & 4 & 140 & 710 & 284.0 & $\mathrm{P}$ & Bio/pmag & \\
\hline & DSDP & 202 & $1237 \mathrm{C}$ & 2.5 & 1 & 6 & 65 & 815 & 326.0 & $\mathrm{~L}$ & Bio/pmag & \\
\hline & DSDP & 202 & $1237 \mathrm{C}$ & 2.5 & 3 & 1 & 58 & 1938 & 775.2 & $\mathrm{P}$ & Bio/pmag & \\
\hline & DSDP & 202 & $1237 \mathrm{C}$ & 2.5 & 3 & 1 & 100 & 1980 & 792.0 & disperse & Bio/pmag & \\
\hline & DSDP & 202 & $1237 \mathrm{D}$ & 2.5 & 2 & 6 & 19 & 1999 & 799.6 & $\mathrm{~L}$ & Bio/pmag & \\
\hline & DSDP & 202 & $1237 \mathrm{~B}$ & 2.5 & 3 & 4 & 57 & 2010 & 804.0 & L & Bio/pmag & \\
\hline & DSDP & 202 & $1237 \mathrm{D}$ & 2.5 & 2 & 6 & 100 & 2080 & 832.0 & L & Bio/pmag & \\
\hline & DSDP & 202 & $1237 \mathrm{C}$ & 2.5 & 3 & 2 & 140 & 2170 & 868.0 & $\mathrm{~L}(\mathrm{P})$ & Bio/pmag & \\
\hline & DSDP & 202 & $1237 \mathrm{C}$ & 2.5 & 3 & 3 & 38 & 2218 & 887.2 & $\mathrm{~L}$ & Bio/pmag & \\
\hline & DSDP & 202 & $1237 \mathrm{~B} / \mathrm{C}$ & 2.5 & 3 & 4 & 120 & 2450 & 980.0 & L & Bio/pmag & \\
\hline & DSDP & 202 & $1237 \mathrm{~B}$ & 2.5 & 4 & 1 & 34 & 2484 & 993.6 & L & Bio/pmag & \\
\hline
\end{tabular}

\begin{tabular}{|c|c|c|c|c|c|c|c|c|c|c|c|}
\hline \multirow[t]{26}{*}{ Equador } & DSDP & 202 & $1238 \mathrm{~A} / 1239 \mathrm{~A}$ & 5.5 & 1 & 1 & 75 & 75 & 13.6 & disperse & Bio/pmag \\
\hline & DSDP & 202 & $1239 \mathrm{~A}$ & 5.5 & 2 & 1 & 75 & 415 & 75.5 & dispers & Bio/pmag \\
\hline & DSDP & 202 & $1239 \mathrm{~A}$ & 5.5 & 2 & 2 & 15 & 505 & 91.8 & $\mathrm{P}$ & Bio/pmag \\
\hline & DSDP & 202 & $1239 \mathrm{~A}$ & 5.5 & 2 & 3 & 30 & 670 & 121.8 & $\mathrm{~L}$ & Bio/pmag \\
\hline & DSDP & 202 & $1239 \mathrm{~A}$ & 5.5 & 2 & 3 & 50 & 690 & 125.5 & $\mathrm{~L}$ & Bio/pmag \\
\hline & DSDP & 202 & $1239 \mathrm{~A}$ & 5.5 & 2 & 3 & 125 & 765 & 139.1 & dispers & Bio/pmag \\
\hline & DSDP & 202 & $1238 \mathrm{C}$ & 5.5 & 3 & 1 & 20 & 1070 & 194.5 & $\mathrm{~L}$ & Bio/pmag \\
\hline & DSDP & 202 & $1239 \mathrm{~B}$ & 5.5 & 2 & 3 & 27 & 1107 & 201.3 & $\mathrm{P}$ & Bio/pmag \\
\hline & DSDP & 202 & $1238 \mathrm{~B} / \mathrm{C}$ & 5.5 & 2 & 3 & 41 & 1141 & 207.5 & $\mathrm{~L}$ & Bio/pmag \\
\hline & DSDP & 202 & $1238 \mathrm{~A}$ & 5.5 & 2 & 6 & 4 & 1258 & 228.7 & $\mathrm{~L}$ & Bio/pmag \\
\hline & DSDP & 202 & 1239B & 5.5 & 2 & 4 & 98 & 1328 & 241.5 & $\mathrm{~L}$ & Bio/pmag \\
\hline & DSDP & 202 & $1239 \mathrm{~B}$ & 5.5 & 2 & 4 & 104 & 1334 & 242.5 & $\mathrm{P}$ & Bio/pmag \\
\hline & DSDP & 202 & $1239 \mathrm{~A}$ & 5.5 & 3 & 4 & 78 & 1818 & 330.5 & $\mathrm{~L}$ & Bio/pmag \\
\hline & DSDP & 202 & $1238 \mathrm{C}$ & 5.5 & 4 & 2 & 140 & 2290 & 416.4 & $\mathrm{~L}$ & Bio/pmag \\
\hline & DSDP & 202 & $1238 \mathrm{C}$ & 5.5 & 4 & 3 & 50 & 2350 & 427.3 & $\mathrm{~L}$ & Bio/pmag \\
\hline & DSDP & 202 & $1238 \mathrm{~B}$ & 5.5 & 3 & 6 & 10 & 2510 & 456.4 & $\mathrm{~L}$ & Bio/pmag \\
\hline & DSDP & 202 & $1238 \mathrm{~B}$ & 5.5 & 3 & 6 & 74 & 2574 & 468.0 & $\mathrm{~L}$ & Bio/pmag \\
\hline & DSDP & 202 & $1238 \mathrm{~B}$ & 5.5 & 3 & 6 & 98 & 2598 & 472.4 & $\mathrm{~L}$ & Bio/pmag \\
\hline & DSDP & 202 & $1238 \mathrm{~A}$ & 5.5 & 4 & 4 & 100 & 2873 & 522.4 & $\mathrm{~L}$ & Bio/pmag \\
\hline & DSDP & 202 & $1238 \mathrm{C}$ & 5.5 & 4 & 7 & 52 & 2952 & 536.7 & $\mathrm{~L}$ & Bio/pmag \\
\hline & DSDP & 202 & $1238 \mathrm{~B} / 1239 \mathrm{~A}$ & 5.5 & 5 & 1 & 75 & 3265 & 593.6 & $\mathrm{~L}$ & Bio/pmag \\
\hline & DSDP & 202 & $1239 \mathrm{~A}$ & 5.5 & 6 & 3 & 48 & 4488 & 816.0 & $\mathrm{~L}$ & Bio/pmag \\
\hline & DSDP & 202 & $1239 \mathrm{~A}$ & 5.5 & 6 & 5 & 80 & 4820 & 876.4 & $\mathrm{~L}$ & Bio/pmag \\
\hline & DSDP & 202 & 1239B & 5.5 & 6 & 3 & 43 & 4923 & 895.1 & $\mathrm{~L}$ & Bio/pmag \\
\hline & DSDP & 202 & $1239 \mathrm{~A}$ & 5.5 & 7 & 1 & 60 & 5150 & 936.4 & $\mathrm{~L}$ & Bio/pmag \\
\hline & DSDP & 202 & $1238 \mathrm{~B} / \mathrm{C}$ & 5.5 & 6 & 5 & 96 & 5296 & 962.9 & $\mathrm{~L}$ & Bio/pmag \\
\hline
\end{tabular}




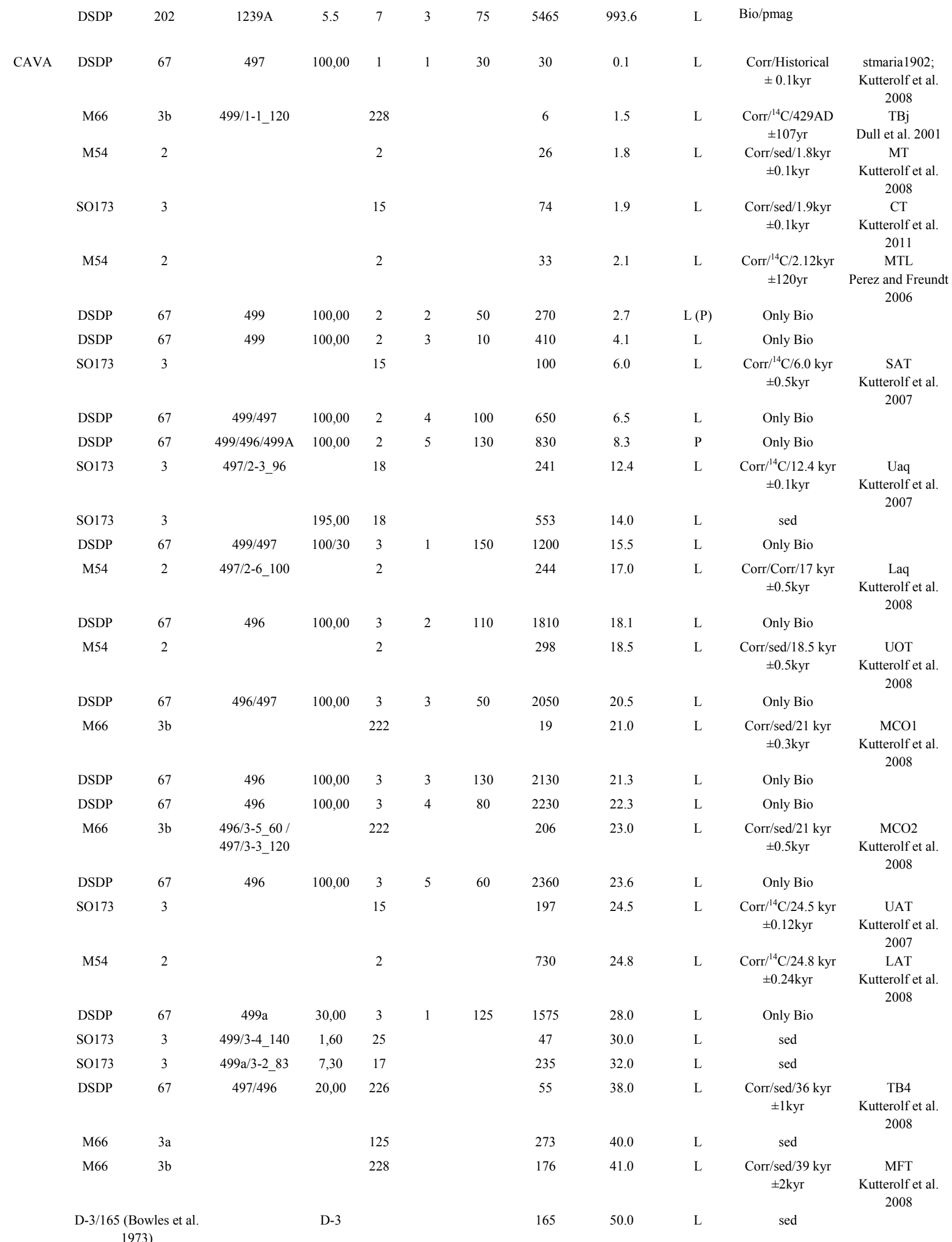




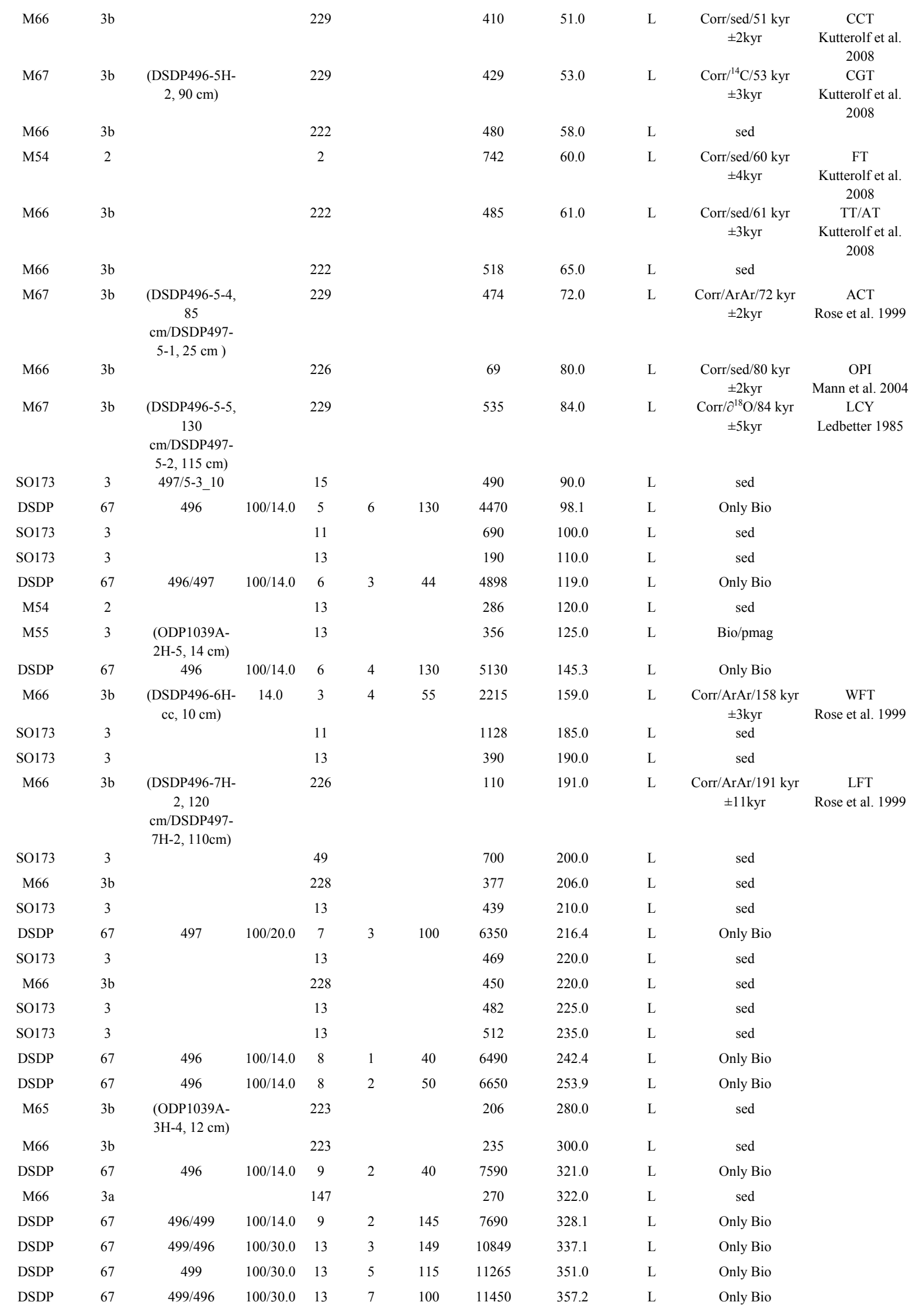




\begin{tabular}{|c|c|c|c|c|c|c|c|c|c|c|c|c|}
\hline & SO173 & 3 & & & 25 & & & 284 & 360.0 & $\mathrm{~L}$ & sed & \\
\hline & DSDP & 67 & $499 / 496$ & $100 / 30.0$ & 14 & 2 & 35 & 11685 & 365.0 & $\mathrm{~L}$ & Only Bio & \\
\hline & M66 & $3 b$ & & & 230 & & & 473 & 402.0 & $\mathrm{~L}$ & sed & \\
\hline & DSDP & 67 & 496 & $100 / 14.0$ & 10 & 4 & 145 & 8945 & 417.8 & $\mathrm{~L}$ & Only Bio & \\
\hline & SO173 & 3 & & & 98 & & & 421 & 450.0 & $\mathrm{~L}$ & sed & \\
\hline & DSDP & 67 & $496 / 497$ & $100 / 14.0$ & 11 & 1 & 100 & 9400 & 450.3 & $\mathrm{~L}$ & Only Bio & \\
\hline & DSDP & 67 & 499 & $100 / 30.0$ & 18 & 1 & 50 & 15350 & 487.2 & L & Only Bio & \\
\hline & SO173 & 3 & & & 98 & & & 454 & 490.0 & $\mathrm{~L}$ & sed & \\
\hline & ODP & & $1043 \mathrm{~A}$ & & 17 & 1 & 142 & 14932 & 500.0 & $\mathrm{~L}$ & Bio/pmag & Clift et al 2005 \\
\hline & ODP & & 1039B & & 7 & 2 & 118 & 5218 & 510.0 & $\mathrm{~L}$ & Bio/pmag & Clift et al 2005 \\
\hline & DSDP & 67 & 499 & $100 / 30.0$ & 18 & 6 & 20 & 16070 & 511.2 & $\mathrm{~L}$ & Only Bio & \\
\hline & DSDP & 67 & 499 & $100 / 30.0$ & 18 & 6 & 150 & 16200 & 515.5 & $\mathrm{~L}$ & Only Bio & \\
\hline & ODP & & 1039B & & 7 & 2 & 122 & 5220 & 540.0 & $\mathrm{~L}$ & Bio/pmag & Clift et al 2005 \\
\hline & DSDP & 67 & 499 & $100 / 30.0$ & 19 & 6 & 80 & 17080 & 545.0 & $\mathrm{~L}$ & Only Bio & \\
\hline & ODP & & $1043 \mathrm{~A}$ & & 19 & 3 & 13 & 17023 & 550.0 & $\mathrm{~L}$ & Bio/pmag & Clift et al 2005 \\
\hline & DSDP & 67 & 499 & $100 / 30.0$ & 22 & 5 & 50 & 19750 & 634.0 & $\mathrm{~L}$ & Only Bio & \\
\hline & DSDP & 67 & 499 & $100 / 30.0$ & 22 & 6 & 80 & 19930 & 640.0 & $\mathrm{~L}$ & Only Bio & \\
\hline & DSDP & 67 & 499 & $100 / 30.0$ & 22 & 6 & 30 & 19980 & 642.0 & $\mathrm{~L}$ & Only Bio & \\
\hline & DSDP & 67 & 497 & $100 / 14.0$ & 13 & 4 & 100 & 11700 & 701.4 & $\mathrm{~L}(\mathrm{P})$ & Only Bio & \\
\hline & ODP & & 1039B & & 8 & 4 & 65 & 6415 & 760.0 & $\mathrm{~L}$ & Bio/pmag & Clift et al 2005 \\
\hline & DSDP & 67 & $496 / 497$ & $100 / 14.0$ & 17 & 1 & 80 & 15080 & 856.0 & $\mathrm{~L}$ & Only Bio & \\
\hline & ODP & & 1039B & & 9 & 1 & 52 & 6902 & 870.0 & $\mathrm{~L}$ & Bio/pmag & Clift et al 2005 \\
\hline & DSDP & 67 & 496 & $100 / 14.0$ & 19 & 1 & 100 & 17000 & 993.1 & $\mathrm{~L}$ & Only Bio & \\
\hline \multirow[t]{14}{*}{ Alaska } & ODP & 18 & $178 / 179$ & 10.5 & 1 & 1 & 108 & 108 & 10.3 & $\mathrm{~L}$ & Bio only & \\
\hline & ODP & 18 & 178 & 10.5 & 1 & 3 & 96 & 391 & 37.2 & $\mathrm{~L}$ & Bio only & \\
\hline & ODP & 18 & 178 & 10.5 & 1 & 4 & 12 & 462 & 44.0 & $\mathrm{~L}$ & Bio only & \\
\hline & ODP & 18 & 179 & 6 & 2 & 2 & 16 & 416 & 86.0 & $\mathrm{~L}$ & Bio only & \\
\hline & ODP & 18 & 179 & 6 & 2 & 2 & 70 & 570 & 95.0 & $\mathrm{~L}$ & Bio only & \\
\hline & ODP & 18 & 178 & 10.5 & 3 & 3 & 20 & 1820 & 173.3 & $\mathrm{~L}$ & Bio only & \\
\hline & ODP & 18 & 178 & 10.5 & 3 & 3 & 144 & 1944 & 185.1 & $\mathrm{~L}$ & Bio only & \\
\hline & ODP & 18 & 178 & 10.5 & 3 & 4 & 187 & 2087 & 198.8 & $\mathrm{~L}$ & Bio only & \\
\hline & ODP & 18 & 178 & 10.5 & 5 & 4 & 6 & 3756 & 357.7 & $\mathrm{~L}$ & Bio only & \\
\hline & ODP & 18 & 179 & 6 & 4 & 2 & 100 & 2500 & 416.7 & $\mathrm{~L}$ & Bio only & \\
\hline & ODP & 18 & 178 & 10.5 & 6 & 4 & 90 & 4740 & 451.4 & $\mathrm{~L}$ & Bio only & \\
\hline & \multicolumn{4}{|c|}{ no recovery $51-60 \mathrm{~m}(460-650 \mathrm{ka})$} & & & & & & & & \\
\hline & ODP & 18 & 178 & 10.5 & 10 & 2 & 119 & 8059 & 767.5 & $\mathrm{~L}$ & Bio only & \\
\hline & ODP & 18 & $178 / 179$ & 10.5 & 12 & 3 & 50 & 9950 & 947.6 & $\mathrm{~L}$ & Bio only & \\
\hline \multirow{12}{*}{$\begin{array}{l}\text { Aleutian } \\
\text { Arc }\end{array}$} & ODP & 19 & 183 & 4.8 & 1 & 2 & 48 & 198 & 41.1 & $\mathrm{~L}$ & Only Bio & \\
\hline & ODP & 19 & 183 & 4.8 & 2 & 2 & 49 & 499 & 103.5 & $\mathrm{~L}$ & Only Bio & \\
\hline & \multicolumn{11}{|c|}{ no recovery/break but abundant glass in silt and clay matrix of chunked recovery; } & \\
\hline & ODP & 19 & 183 & 4.8 & 4 & 1 & 120 & 2220 & 460.4 & $\mathrm{~L}$ & Only Bio & \\
\hline & ODP & 19 & 183 & 4.8 & 4 & 1 & 141 & 2241 & 464.8 & $\mathrm{~L}$ & Only Bio & \\
\hline & ODP & 19 & 183 & 4.8 & 4 & 3 & 12 & 2412 & 500.3 & $\mathrm{~L}$ & Only Bio & \\
\hline & ODP & 19 & 183 & 4.8 & 4 & 3 & 82 & 2482 & 514.8 & $\mathrm{~L}$ & Only Bio & \\
\hline & ODP & 19 & 183 & 4.8 & 4 & 4 & 84 & 2634 & 546.3 & $\mathrm{~L}$ & Only Bio & \\
\hline & ODP & 19 & 183 & 4.8 & 5 & 1 & 95 & 3095 & 641.9 & $\mathrm{~L}$ & Only Bio & \\
\hline & ODP & 19 & 183 & 4.8 & 5 & 1 & 130 & 3130 & 649.2 & $\mathrm{~L}$ & Only Bio & \\
\hline & ODP & 19 & 183 & 4.8 & 5 & 1 & 140 & 3146 & 652.5 & $\mathrm{~L}$ & Only Bio & \\
\hline & ODP & 19 & 183 & 4.8 & 5 & 2 & 80 & 3230 & 669.9 & $\mathrm{~L}$ & Only Bio & \\
\hline
\end{tabular}




\begin{tabular}{|c|c|c|c|c|c|c|c|c|c|c|c|}
\hline & ODP & 19 & 183 & 4.8 & 5 & 2 & 150 & 3250 & 674.1 & $\mathrm{~L}$ & Only Bio \\
\hline & ODP & 19 & 183 & 4.8 & 5 & 5 & 41 & 3641 & 755.2 & $\mathrm{~L}$ & Only Bio \\
\hline & ODP & 19 & 183 & 4.8 & 6 & 1 & 124 & 4024 & 834.6 & $\mathrm{~L}$ & Only Bio \\
\hline & ODP & 19 & 183 & 4.8 & 6 & 3 & 120 & 4420 & 916.7 & $\mathrm{~L}$ & Only Bio \\
\hline & ODP & 19 & 183 & 4.8 & 6 & 5 & 136 & 4730 & 981.0 & $\mathrm{~L}$ & Only Bio \\
\hline \multirow{14}{*}{$\begin{array}{l}\text { Aleutian } \\
\text { Basin }\end{array}$} & ODP & 19 & 190 & 9.3 & 2 & 2 & 96 & 846 & 91.0 & $\mathrm{~L}$ & Only Bio \\
\hline & ODP & 19 & 190 & 9.3 & 2 & $\mathrm{cc}$ & 315 & 915 & 98.4 & $\mathrm{~L}$ & Only Bio \\
\hline & ODP & 19 & 190 & 9.3 & 3 & 1 & 136 & 1636 & 175.9 & $\mathrm{~L}$ & Only Bio \\
\hline & ODP & 19 & 190 & 9.3 & 3 & 2 & 22 & 1672 & 179.8 & $\mathrm{~L}$ & Only Bio \\
\hline & ODP & 19 & 190 & 9.3 & 3 & 2 & 48 & 1698 & 182.6 & $\mathrm{~L}$ & Only Bio \\
\hline & ODP & 19 & 190 & 9.3 & 4 & 2 & 70 & 2620 & 281.7 & $\mathrm{~L}$ & Only Bio \\
\hline & ODP & 19 & 190 & 9.3 & 4 & 3 & 7 & 2707 & 291.1 & $\mathrm{~L}$ & Only Bio \\
\hline & ODP & 19 & 190 & 9.3 & 4 & 3 & 105 & 2805 & 301.6 & $\mathrm{~L}$ & Only Bio \\
\hline & ODP & 19 & 190 & 9.3 & 6 & 5 & 110 & 5010 & 538.7 & $\mathrm{~L}$ & Only Bio \\
\hline & ODP & 19 & 190 & 9.3 & 6 & $\mathrm{cc}$ & 155 & 5055 & 543.5 & $\mathrm{~L}$ & Only Bio \\
\hline & ODP & 19 & 190 & 9.3 & 7 & 1 & 70 & 7572 & 814.2 & $\mathrm{~L}$ & Only Bio \\
\hline & ODP & 19 & 190 & 9.3 & 7 & 5 & 10 & 8110 & 872.0 & $\mathrm{~L}$ & Only Bio \\
\hline & ODP & 19 & 190 & 9.3 & 8 & 2 & 91 & 8641 & 929.1 & $\mathrm{~L}$ & Only Bio \\
\hline & ODP & 19 & 190 & 9.3 & 8 & 2 & 145 & 8695 & 934.9 & $\mathrm{~L}$ & Only Bio \\
\hline \multirow{27}{*}{$\begin{array}{c}\text { Kamtchatk } \\
\text { a }\end{array}$} & ODP & 19 & 192 & 4.1 & 1 & 1 & 121 & 121 & 29.5 & $\mathrm{~L}$ & Only Bio \\
\hline & ODP & 19 & 192 & 4.1 & 1 & 1 & 142 & 142 & 34.6 & $\mathrm{~L}$ & Only Bio \\
\hline & ODP & 19 & 192 & 4.1 & 1 & $\mathrm{cc}$ & $\mathrm{cc}$ & 170 & 41.4 & $\mathrm{~L}$ & Only Bio \\
\hline & ODP & 19 & 192 & 4.1 & 2 & 1 & 148 & 248 & 60.4 & $\mathrm{~L}$ & Only Bio \\
\hline & ODP & 19 & 192 & 4.1 & 2 & 4 & 82 & 632 & 153.9 & $\mathrm{~L}$ & Only Bio \\
\hline & ODP & 19 & 192 & 4.1 & 2 & 4 & 140 & 692 & 168.5 & $\mathrm{~L}$ & Only Bio \\
\hline & ODP & 19 & 192 & 4.1 & 3 & 1 & 120 & 1120 & 272.7 & $\mathrm{~L}$ & Only Bio \\
\hline & ODP & 19 & 192 & 4.1 & 3 & 2 & 60 & 1210 & 294.6 & $\mathrm{~L}$ & Only Bio \\
\hline & ODP & 19 & 192 & 4.1 & 3 & 2 & 88 & 1238 & 301.4 & $\mathrm{~L}$ & Only Bio \\
\hline & ODP & 19 & 192 & 4.1 & 3 & 2 & 143 & 1293 & 314.8 & $\mathrm{~L}$ & Only Bio \\
\hline & ODP & 19 & 192 & 4.1 & 3 & 3 & 48 & 1348 & 328.2 & $\mathrm{~L}$ & Only Bio \\
\hline & ODP & 19 & 192 & 4.1 & 3 & 3 & 150 & 1450 & 353.1 & $\mathrm{~L}$ & Only Bio \\
\hline & ODP & 19 & 192 & 4.1 & 4 & 1 & 5 & 1905 & 463.8 & $\mathrm{~L}$ & Only Bio \\
\hline & ODP & 19 & 192 & 4.1 & 4 & 1 & 147 & 2047 & 498.4 & $\mathrm{~L}$ & Only Bio \\
\hline & ODP & 19 & 192 & 4.1 & 4 & 2 & 112 & 2162 & 526.4 & $\mathrm{~L}$ & Only Bio \\
\hline & ODP & 19 & 192 & 4.1 & 4 & 2 & 130 & 2180 & 530.8 & $\mathrm{~L}$ & Only Bio \\
\hline & ODP & 19 & 192 & 4.1 & 4 & 3 & 60 & 2260 & 550.3 & $\mathrm{~L}$ & Only Bio \\
\hline & ODP & 19 & 192 & 4.1 & 4 & 4 & 55 & 2405 & 585.6 & $\mathrm{~L}$ & Only Bio \\
\hline & ODP & 19 & 192 & 4.1 & 4 & 6 & 81 & 2781 & 677.1 & $\mathrm{~L}$ & Only Bio \\
\hline & ODP & 19 & 192 & 4.1 & 4 & 6 & 149 & 2799 & 681.5 & $\mathrm{~L}$ & Only Bio \\
\hline & ODP & 19 & 192 & 4.1 & 5 & 2 & 18 & 2968 & 722.7 & $\mathrm{~L}$ & Only Bio \\
\hline & ODP & 19 & 192 & 4.1 & 5 & 2 & 73 & 3023 & 736.1 & $\mathrm{~L}$ & Only Bio \\
\hline & ODP & 19 & 192 & 4.1 & 5 & 3 & 123 & 3223 & 784.8 & $\mathrm{~L}$ & Only Bio \\
\hline & ODP & 19 & 192 & 4.1 & 5 & 4 & 73 & 3323 & 809.1 & $\mathrm{~L}$ & Only Bio \\
\hline & ODP & 19 & 192 & 4.1 & 5 & 4 & 94 & 3344 & 814.2 & $\mathrm{~L}$ & Only Bio \\
\hline & ODP & 19 & 192 & 4.1 & 5 & 5 & 131 & 3531 & 859.8 & $\mathrm{~L}$ & Only Bio \\
\hline & ODP & 19 & 192 & 4.1 & 5 & cc & 165 & 3565 & 868.0 & $\mathrm{~L}$ & Only Bio \\
\hline $\begin{array}{c}\text { Nankai } \\
\text { Through }\end{array}$ & DSDP & 190 & 1173 & 38.5 & 1 & 1 & 15 & 15 & 0.4 & $\mathrm{~L}$ & Bio/pmag \\
\hline
\end{tabular}




\begin{tabular}{|c|c|c|c|c|c|c|c|c|c|c|}
\hline DSDP & 190 & $1174 \mathrm{~A}$ & 70.9 & 1 & 3 & 36 & 336 & 4.7 & $\mathrm{~L}$ & Bio/pmag \\
\hline DSDP & 190 & $1173 / 1174 \mathrm{~A}$ & 38.5 & 1 & 2 & 70 & 220 & 5.7 & disperse & Bio/pmag \\
\hline DSDP & 190 & 1173 & 38.5 & 1 & 5 & 27 & 627 & 16.3 & disperse & Bio/pmag \\
\hline DSDP & 190 & 1173 & 38.5 & 2 & 1 & 113 & 827 & 21.5 & disperse & Bio/pmag \\
\hline DSDP & 190 & $1174 \mathrm{~A}$ & 70.9 & 4 & 4 & 100 & 2890 & 40.8 & $\mathrm{~L}$ & Bio/pmag \\
\hline DSDP & 190 & 1173 & 38.5 & 5 & 1 & 38 & 3602 & 93.6 & $\mathrm{~L}$ & Bio/pmag \\
\hline DSDP & 190 & 1173 & 38.5 & 5 & 6 & 117 & 4431 & 115.1 & $\mathrm{~L}$ & Bio/pmag \\
\hline DSDP & 190 & 1173 & 38.5 & 6 & 2 & 44 & 4708 & 122.3 & disperse & Bio/pmag \\
\hline DSDP & 190 & 1173 & 38.5 & 7 & 1 & 111 & 5575 & 144.8 & $\mathrm{~L}$ & Bio/pmag \\
\hline DSDP & 190 & 1173 & 38.5 & 7 & 4 & 15 & 5929 & 154.0 & $\mathrm{~L}$ & Bio/pmag \\
\hline DSDP & 190 & 1173 & 38.5 & 7 & 4 & 37 & 5951 & 154.6 & $\mathrm{~L}$ & Bio/pmag \\
\hline DSDP & 190 & 1173 & 38.5 & 8 & 5 & 70 & 7084 & 184.0 & disperse & Bio/pmag \\
\hline DSDP & 190 & 1173 & 38.5 & 9 & 1 & 101 & 7465 & 193.9 & $\mathrm{~L}$ & Bio/pmag \\
\hline DSDP & 190 & 1173 & 38.5 & 10 & 2 & 92 & 8556 & 222.2 & $\mathrm{~L}$ & Bio/pmag \\
\hline DSDP & 190 & 1173 & 38.5 & 10 & 3 & 2 & 8616 & 223.8 & $\mathrm{~L}$ & Bio/pmag \\
\hline DSDP & 190 & 1173 & 38.5 & 10 & 4 & 72 & 8836 & 229.5 & $\mathrm{~L}$ & Bio/pmag \\
\hline DSDP & 190 & 1173 & 38.5 & 10 & 6 & 135 & 9205 & 239.1 & $\mathrm{~L}$ & Bio/pmag \\
\hline DSDP & 190 & 1173/1174B & 38.5 & 11 & 5 & 121 & 9985 & 259.4 & $\mathrm{~L}$ & Bio/pmag \\
\hline DSDP & 190 & 1173 & 38.5 & 12 & 2 & 134 & 10498 & 272.7 & $\mathrm{~L}$ & Bio/pmag \\
\hline DSDP & 190 & 1173 & 38.5 & 12 & 4 & 111 & 10775 & 279.9 & $\mathrm{~L}$ & Bio/pmag \\
\hline DSDP & 190 & 1173 & 38.5 & 12 & 4 & 125 & 10789 & 280.2 & $\mathrm{~L}$ & Bio/pmag \\
\hline DSDP & 190 & 1173 & 38.5 & 12 & 6 & 9 & 10973 & 285.0 & $\mathrm{~L}$ & Bio/pmag \\
\hline DSDP & 190 & 1174B & 70.9 & 10 & 1 & 14 & 22734 & 320.6 & $\mathrm{~L}$ & Bio/pmag \\
\hline DSDP & 190 & 1173/1174B & 9.2 & 13 & 3 & 145 & 11609 & 352.2 & disperse & Bio/pmag \\
\hline DSDP & 190 & 1174B & 70.9 & 13 & 1 & 137 & 25757 & 363.3 & $\mathrm{~L}$ & Bio/pmag \\
\hline DSDP & 190 & 1174B & 70.9 & 17 & 1 & 115 & 29575 & 417.1 & $\mathrm{~L}$ & Bio/pmag \\
\hline DSDP & 190 & 1173/1174B & 9.2 & 14 & 3 & 106 & 12520 & 451.2 & $\mathrm{~L}$ & Bio/pmag \\
\hline DSDP & 190 & 1173 & 9.2 & 15 & 1 & 143 & 13207 & 525.9 & disperse & Bio/pmag \\
\hline DSDP & 190 & 1174B & 70.9 & 27 & 1 & 80 & 39130 & 551.9 & $\mathrm{~L}$ & Bio/pmag \\
\hline DSDP & 190 & 1174B & 70.9 & 27 & 2 & 91 & 39291 & 554.2 & $\mathrm{~L}$ & Bio/pmag \\
\hline DSDP & 190 & 1174B & 70.9 & 28 & 1 & 112 & 40132 & 566.0 & $\mathrm{~L}$ & Bio/pmag \\
\hline DSDP & 190 & 1174B & 70.9 & 31 & 2 & 107 & 43157 & 608.7 & $\mathrm{~L}$ & Bio/pmag \\
\hline DSDP & 190 & 1173/1174B & 70.9 & 31 & 3 & 124 & 43324 & 611.1 & $\mathrm{~L}$ & Bio/pmag \\
\hline DSDP & 190 & 1173/1174B & 9.2 & 16 & 2 & 90 & 14254 & 639.7 & $\mathrm{~L}$ & Bio/pmag \\
\hline DSDP & 190 & 1174 & 70.9 & 34 & 2 & 34 & 45974 & 648.4 & $\mathrm{~L}$ & Bio/pmag \\
\hline DSDP & 190 & 1174 & 70.9 & 34 & 3 & 89 & 46179 & 651.3 & $\mathrm{~L}$ & Bio/pmag \\
\hline DSDP & 190 & 1174B & 70.9 & 37 & 2 & 125 & 48845 & 688.9 & $\mathrm{~L}$ & Bio/pmag \\
\hline DSDP & 190 & 1174B & 70.9 & 37 & 2 & 133 & 48853 & 689.0 & $\mathrm{~L}$ & Bio/pmag \\
\hline DSDP & 190 & 1174B & 70.9 & 37 & 3 & 8 & 48878 & 689.4 & $\mathrm{~L}$ & Bio/pmag \\
\hline DSDP & 190 & 1173 & 9.2 & 16 & 5 & 113 & 14727 & 691.1 & $\mathrm{~L}$ & Bio/pmag \\
\hline DSDP & 190 & 1174 & 70.9 & 37 & 6 & 42 & 49362 & 696.2 & $\mathrm{~L}$ & Bio/pmag \\
\hline DSDP & 190 & 1174 & 70.9 & 38 & 3 & 64 & 49894 & 703.7 & $\mathrm{~L}$ & Bio/pmag \\
\hline DSDP & 190 & 1174 & 70.9 & 39 & 1 & 0 & 50490 & 712.1 & $\mathrm{~L}$ & Bio/pmag \\
\hline DSDP & 190 & 1174 & 70.9 & 39 & 4 & 39 & 50979 & 719.0 & $\mathrm{~L}$ & Bio/pmag \\
\hline DSDP & 190 & 1174 & 70.9 & 39 & 4 & 65 & 51005 & 719.4 & $\mathrm{~L}$ & Bio/pmag \\
\hline DSDP & 190 & 1173/1174B & 9.2 & 17 & 1 & 50 & 15014 & 722.3 & $\mathrm{~L}$ & Bio/pmag \\
\hline DSDP & 190 & 1174 & 70.9 & 40 & 3 & 125 & 51885 & 731.8 & $\mathrm{~L}$ & Bio/pmag \\
\hline DSDP & 190 & 1174 & 70.9 & 41 & 1 & 103 & 52533 & 740.9 & $\mathrm{~L}$ & Bio/pmag \\
\hline DSDP & 190 & 1173/1174B & 9.2 & 17 & 3 & 50 & 15314 & 754.9 & $\mathrm{~L}$ & Bio/pmag \\
\hline DSDP & 190 & 1174 & 70.9 & 42 & 2 & 112 & 53652 & 756.7 & $\mathrm{~L}$ & Bio/pmag \\
\hline DSDP & 190 & 1173/1174B & 9.2 & 18 & 4 & 85 & 16449 & 878.3 & $\mathrm{~L}$ & Bio/pmag \\
\hline DSDP & 190 & 1173/1174B & 9.2 & 19 & 1 & 20 & 16884 & 925.6 & $\mathrm{~L}$ & Bio/pmag \\
\hline
\end{tabular}




$\begin{array}{llcllllllll}\text { DSDP } & 190 & 1173 / 1174 \mathrm{~B} & 9.2 & 19 & 1 & 93 & 16957 & 933.5 & \mathrm{~L} & \mathrm{Bio} / \mathrm{pmag} \\ \text { DSDP } & 190 & 1173 / 1174 \mathrm{~B} & 9.2 & 19 & 1 & 96 & 16960 & 933.8 & \mathrm{~L} & \mathrm{Bio} / \mathrm{pmag} \\ \text { DSDP } & 190 & 1173 & 9.2 & 19 & 1 & 97 & 16961 & 933.9 & \mathrm{~L} & \mathrm{Bio} / \mathrm{pmag} \\ \text { DSDP } & 190 & 1173 / 1174 \mathrm{~B} & 9.2 & 19 & 3 & 103 & 17263 & 966.8 & \mathrm{~L} & \mathrm{Bio} / \mathrm{pmag}\end{array}$

New

Zealand

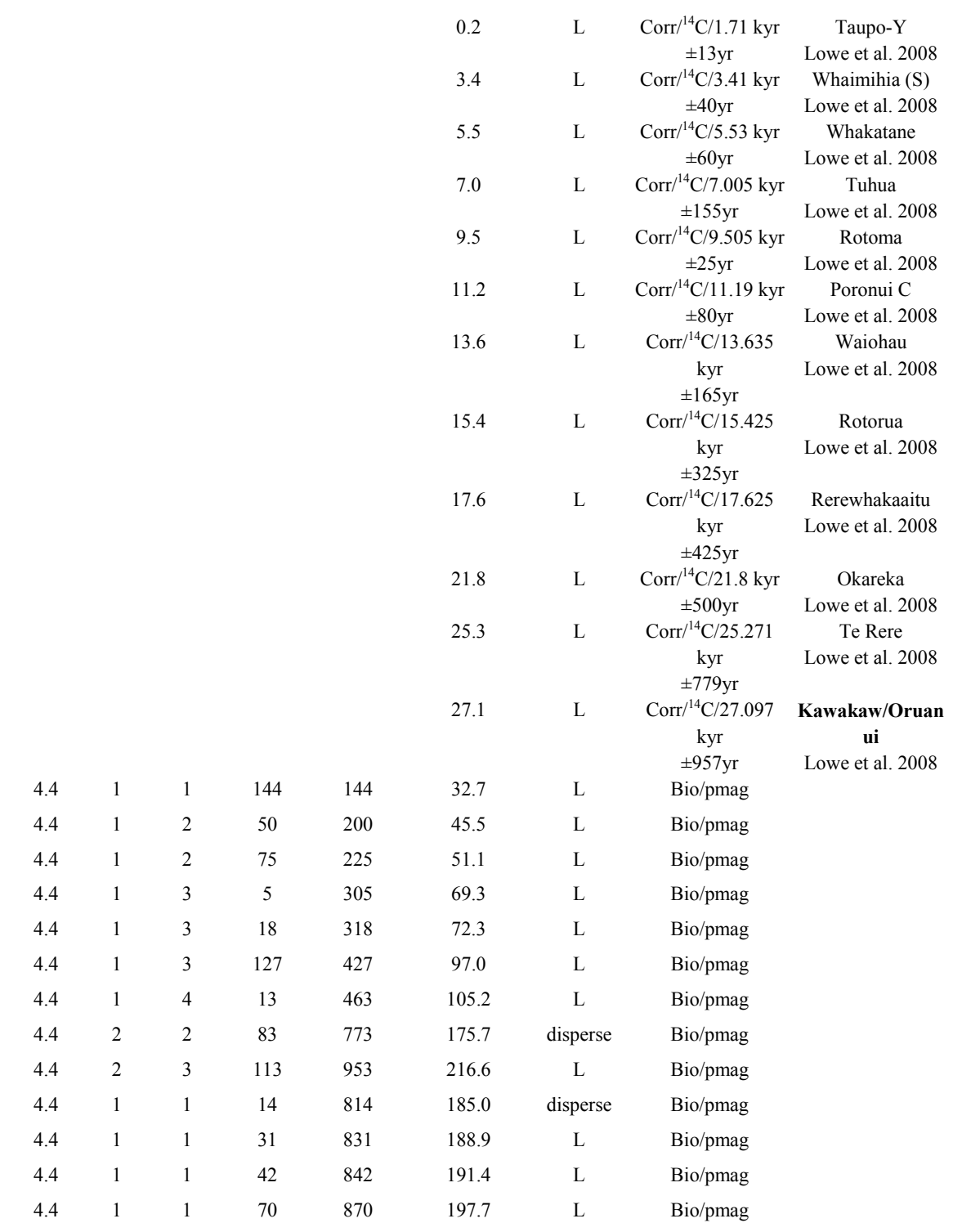

ODP gap: abundant tephra Layers missed through washout during coring

MD 97-2121

MD $\quad 97-2121$

MD $\quad 97-2121$

MD 97-2121

MD 97-2121

MD 97-2121

MD $\quad 97-2121$

MD 97-2121

MD $\quad 97-2121$

MD 97-2121

MD $\quad 97-2121$

MD 97-2121

ODP 181

ODP 181

ODP 181

ODP 181

ODP 181

ODP 181

ODP 181

ODP 181

ODP 181

ODP $\quad 181$

ODP 181

ODP 181

ODP 181

DP 181

ODP 181

ODP 181

ODP 181

ODP 181

ODP 181

ODP 181

ODP 181

ODP 181

ODP 181
$1124 \mathrm{C}$

$1124 \mathrm{C}$

$1124 \mathrm{C}$

$1124 \mathrm{C}$

$1124 \mathrm{C}$

$1124 \mathrm{C}$

$1124 \mathrm{C}$

$1124 \mathrm{C}$

$1124 \mathrm{C}$

4.4

$\begin{array}{lll}4.4 & 2 & 4\end{array}$

$4 \quad 2 \quad 6$

$\begin{array}{lll}4.4 & 2 & 6 \\ 4.4 & 3 & 1\end{array}$

$\begin{array}{lll}4.4 & 3 & 1\end{array}$

$\begin{array}{lll}.4 & 3 & 1 \\ .4 & 3 & 2\end{array}$

$\begin{array}{lll}.4 & 3 & 2 \\ 4 & 3 & 3\end{array}$

$\begin{array}{lll}.4 & 3 & 3 \\ 4.4 & 3 & 4\end{array}$

$\begin{array}{lll}.4 & 3 & 4 \\ 4.4 & 3 & 4\end{array}$

$\begin{array}{lll}4.4 & 3 & 4 \\ 4.4 & 3 & 5\end{array}$ $\begin{array}{ll}70 & 2180 \\ 53 & 2400\end{array}$

$74.5 \quad 2425$

$89 \quad 2800$

$96 \quad 2816$

$85 \quad 2955$

$57 \quad 3077$

$56 \quad 3226$

$110 \quad 3280$

$60 \quad 3380$
495.5
545.5

551.1

636.4

640.0

671.6

699.3

733.2

745.5

768.2

$\begin{array}{cc}\text { L } & \text { Bio/pmag } \\ \text { L } & \text { Bio/pmag } \\ \text { L } & \text { Bio/pmag } \\ \text { L } & \text { Bio/pmag } \\ \text { disperse } & \text { Bio/pmag } \\ \text { L } & \text { Bio/pmag } \\ \text { L } & \text { Bio/pmag } \\ \text { L } & \text { Bio/pmag } \\ \text { L } & \text { Bio/pmag } \\ \text { L } & \text { Bio/pmag }\end{array}$




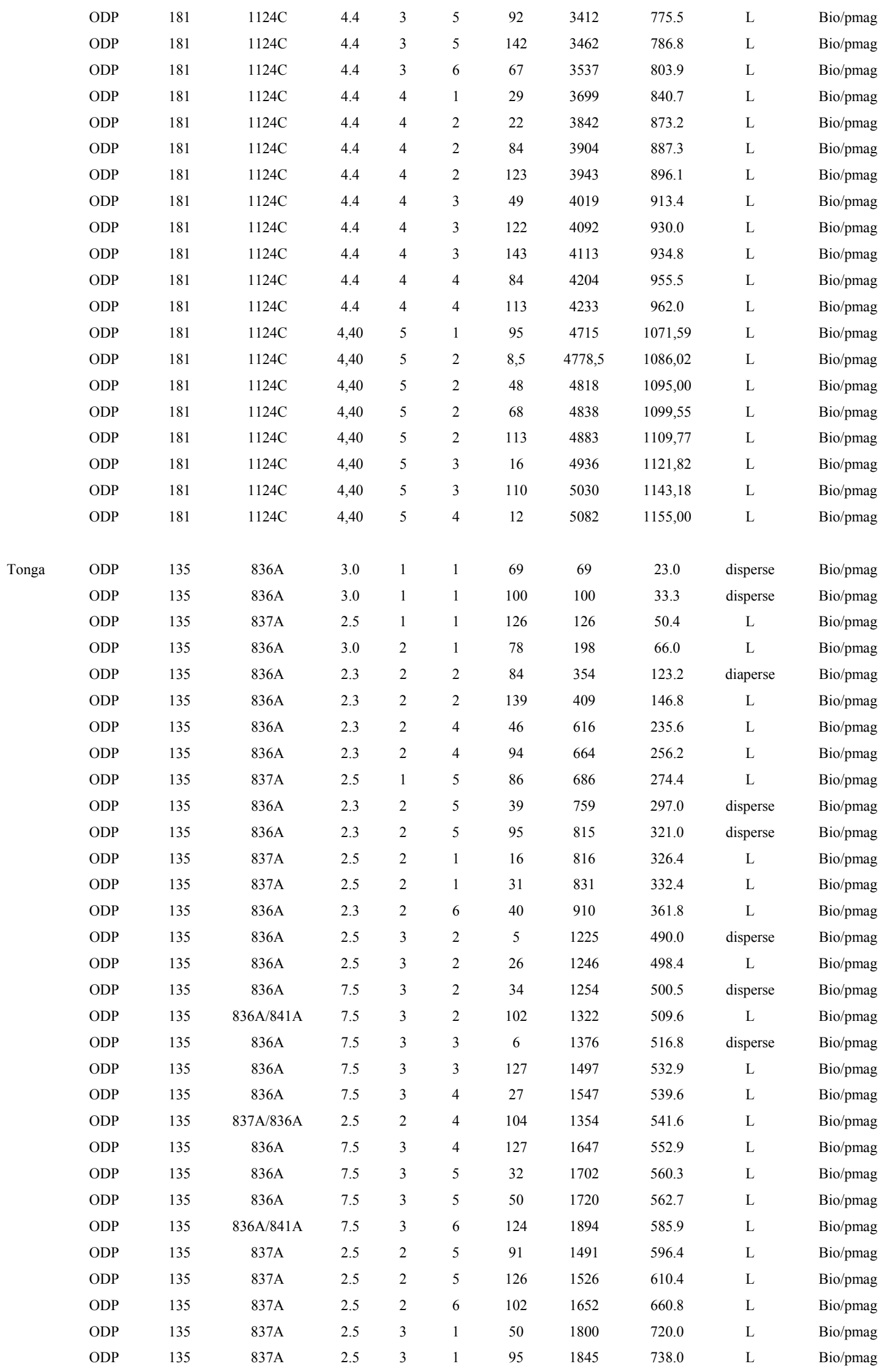




$\begin{array}{lllllllllll}\text { ODP } & 135 & 837 \mathrm{~A} & 2.5 & 3 & 2 & 60 & 1960 & 784.0 & \mathrm{~L} & \text { Bio/pmag } \\ \text { ODP } & 135 & 837 \mathrm{~A} & 5.0 & 3 & 2 & 118 & 2018 & 803.6 & \mathrm{~L} & \mathrm{Bio} / \mathrm{pmag} \\ \text { ODP } & 135 & 837 \mathrm{~A} & 5.0 & 3 & 3 & 22 & 2072 & 814.4 & \mathrm{~L} & \mathrm{Bio} / \mathrm{pmag} \\ \text { ODP } & 135 & 837 \mathrm{~A} & 5.0 & 4 & 1 & 10 & 2710 & 942.0 & \mathrm{~L} & \mathrm{Bio} / \mathrm{pmag} \\ \text { ODP } & 135 & 837 \mathrm{~A} & 5.0 & 4 & 1 & 145 & 2845 & 969.0 & \mathrm{~L} & \mathrm{Bio} / \mathrm{pmag} \\ \text { ODP } & 135 & 837 \mathrm{~A} & 5.0 & 4 & 2 & 124 & 2974 & 994.8 & \mathrm{~L} & \mathrm{Bio} / \mathrm{pmag} \\ \text { ODP } & 135 & 837 \mathrm{~A} & 5,00 & 4 & 3 & 61 & 3061 & 1012,20 & \mathrm{~L} & \mathrm{Bio} / \mathrm{pmag} \\ \text { ODP } & 135 & 837 \mathrm{~A} & 5,00 & 4 & 3 & 84 & 3084 & 1016,80 & \mathrm{~L} & \text { Bio/pmag } \\ \text { ODP } & 135 & 837 \mathrm{~A} & 5,00 & 4 & 3 & 142 & 3142 & 1028,40 & \mathrm{~L} & \mathrm{Bio} / \mathrm{pmag} \\ \text { ODP } & 135 & 837 \mathrm{~A} & 5,00 & 4 & 4 & 95 & 3245 & 1049,00 & \mathrm{~L} & \mathrm{Bio} / \mathrm{pmag} \\ \text { ODP } & 135 & 837 \mathrm{~A} & 5,00 & 4 & 5 & 14 & 3314 & 1062,80 & \mathrm{~L} & \mathrm{Bio} / \mathrm{pmag} \\ \text { ODP } & 135 & 837 \mathrm{~A} & 5,00 & 4 & 6 & 70 & 3520 & 1104,00 & \mathrm{~L} & \mathrm{Bio} / \mathrm{pmag} \\ \text { ODP } & 135 & 837 \mathrm{~A} & 5,00 & 4 & 6 & 84 & 3534 & 1106,80 & \mathrm{~L} & \mathrm{Bio} / \mathrm{pmag} \\ \text { ODP } & 135 & 837 \mathrm{~A} & 5,00 & 4 & \mathrm{cc} & 21 & 3621 & 1124,20 & \mathrm{~L} & \mathrm{Bio} / \mathrm{pmag}\end{array}$

\begin{tabular}{|c|c|c|c|c|c|c|c|c|c|c|c|}
\hline \multirow[t]{35}{*}{ Philippines } & ODP & 124 & $769 \mathrm{~A}$ & 8.6 & 1 & 1 & 40 & 40 & 4.7 & $\mathrm{~L}$ & Bio/pmag \\
\hline & ODP & 124 & $767 \mathrm{~A} / 769 \mathrm{~A}$ & 5.9 & 1 & 1 & 75 & 75 & 12.7 & $\mathrm{~L}$ & Bio/pmag \\
\hline & ODP & 124 & $769 \mathrm{~A}$ & 8.6 & 1 & 2 & 46 & 196 & 22.8 & $\mathrm{~L}$ & Bio/pmag \\
\hline & ODP & 124 & $767 \mathrm{~B} / 770 \mathrm{~B}$ & 5.9 & 1 & 2 & 80 & 230 & 38.9 & $\mathrm{~L}$ & Bio/pmag \\
\hline & ODP & 124 & $768 \mathrm{~A}$ & 10.5 & 1 & 3 & 133 & 433 & 41.2 & disperse & Bio/pmag \\
\hline & ODP & 124 & $768 \mathrm{~B}$ & 10.5 & 2 & 1 & 41 & 441 & 42.0 & L & Bio/pmag \\
\hline & ODP & 124 & $767 \mathrm{~A}$ & 5.9 & 1 & 3 & 73 & 373 & 63.1 & $\mathrm{~L}$ & Bio/pmag \\
\hline & ODP & 124 & $769 \mathrm{~A}$ & 8.6 & 1 & 5 & 20 & 620 & 72.1 & L & Bio/pmag \\
\hline & ODP & 124 & $767 \mathrm{~B} / 769 \mathrm{~A}$ & 5.9 & 1 & 4 & 63 & 513 & 86.8 & disperse & Bio/pmag \\
\hline & ODP & 124 & $770 \mathrm{~B} / 769 \mathrm{~B}$ & 3.6 & 1 & 3 & 77 & 377 & 104.7 & $\mathrm{~L}$ & Bio/pmag \\
\hline & ODP & 124 & $767 \mathrm{~B} / 770 \mathrm{~B}$ & 5.9 & 1 & 5 & 141 & 741 & 125.4 & $\mathrm{~L}$ & Bio/pmag \\
\hline & ODP & 124 & 767B & 5.9 & 1 & 6 & 126 & 876 & 148.2 & $\mathrm{~L}$ & Bio/pmag \\
\hline & ODP & 124 & $767 \mathrm{~B} / 769 \mathrm{~A}$ & 5.9 & 2 & 1 & 68 & 968 & 163.8 & $\mathrm{~L}$ & Bio/pmag \\
\hline & ODP & 124 & $769 \mathrm{~A} / 769 \mathrm{~B}$ & 8.6 & 2 & 5 & 50 & 1490 & 173.3 & $\mathrm{~L}$ & Bio/pmag \\
\hline & ODP & 124 & $769 \mathrm{~A}$ & 8.6 & 2 & 5 & 136 & 1576 & 183.3 & $\mathrm{~L}$ & Bio/pmag \\
\hline & ODP & 124 & 767B/769A & 5.9 & 2 & 2 & 65 & 1115 & 188.7 & $\mathrm{~L}$ & Bio/pmag \\
\hline & ODP & 124 & $769 \mathrm{~A}$ & 8.6 & 2 & 6 & 77 & 1667 & 193.8 & $\mathrm{~L}$ & Bio/pmag \\
\hline & ODP & 124 & $767 \mathrm{~B} / 768 \mathrm{~B}$ & 5.9 & 2 & 2 & 128 & 1178 & 199.3 & $\mathrm{~L}$ & Bio/pmag \\
\hline & ODP & 124 & $769 \mathrm{~A}$ & 8.6 & 2 & 7 & 31 & 1771 & 205.9 & $\mathrm{~L}$ & Bio/pmag \\
\hline & ODP & 124 & $768 \mathrm{~B}$ & 10.5 & 3 & $\mathrm{cc}$ & 19 & 2300 & 219.0 & $\mathrm{~L}$ & Bio/pmag \\
\hline & ODP & 124 & $769 \mathrm{~A}$ & 8.6 & 3 & 3 & 35 & 2125 & 247.1 & $\mathrm{~L}$ & Bio/pmag \\
\hline & ODP & 124 & 767B/769A & 5.9 & 2 & 5 & 135 & 1635 & 276.6 & $\mathrm{~L}$ & Bio/pmag \\
\hline & ODP & 124 & $769 \mathrm{~B}$ & 8.6 & 4 & 1 & 55 & 2495 & 290.1 & $\mathrm{~L}$ & Bio/pmag \\
\hline & ODP & 124 & $769 \mathrm{~B}$ & 8.6 & 4 & 1 & 112 & 2552 & 296.7 & $\mathrm{~L}$ & Bio/pmag \\
\hline & ODP & 124 & $767 \mathrm{~B}$ & 5.9 & 2 & 7 & 40 & 1840 & 311.3 & L & Bio/pmag \\
\hline & ODP & 124 & 767B & 5.9 & 3 & 1 & 90 & 1940 & 328.3 & $\mathrm{~L}$ & Bio/pmag \\
\hline & ODP & 124 & $767 \mathrm{~B}$ & 5.9 & 3 & 1 & 114 & 1964 & 332.3 & $\mathrm{~L}$ & Bio/pmag \\
\hline & ODP & 124 & $767 \mathrm{~B} / 769 \mathrm{~A}$ & 5.9 & 3 & 2 & 50 & 2050 & 346.9 & disperse & Bio/pmag \\
\hline & ODP & 124 & $767 \mathrm{~B} / 769 \mathrm{~A}$ & 5.9 & 3 & 3 & 65 & 2215 & 374.8 & $\mathrm{~L}$ & Bio/pmag \\
\hline & ODP & 124 & $767 \mathrm{~B}$ & 5.9 & 3 & 4 & 30 & 2330 & 394.2 & disperse & Bio/pmag \\
\hline & ODP & 124 & $769 A$ & 8.6 & 4 & 6 & 105 & 3595 & 418.0 & $\mathrm{~L}$ & Bio/pmag \\
\hline & ODP & 124 & $769 \mathrm{~A}$ & 8.6 & 5 & 1 & 105 & 3795 & 441.3 & $\mathrm{~L}$ & Bio/pmag \\
\hline & ODP & 124 & $769 \mathrm{~A} / \mathrm{B}$ & 8.6 & 5 & 2 & 20 & 3860 & 448.8 & $\mathrm{~L}$ & Bio/pmag \\
\hline & ODP & 124 & $767 \mathrm{~B} / 769 \mathrm{~A}$ & 5.9 & 3 & 6 & 60 & 2660 & 450.1 & disperse & Bio/pmag \\
\hline & ODP & 124 & $769 \mathrm{~A} / 768 \mathrm{~B}$ & 8.6 & 5 & 2 & 85 & 3935 & 457.6 & $\mathrm{~L}$ & Bio/pmag \\
\hline & ODP & 124 & $767 \mathrm{~B} / 769 \mathrm{~A}$ & 5.9 & 4 & 2 & 123 & 3077 & 520.6 & disperse & Bio/pmag \\
\hline
\end{tabular}




$\begin{array}{lcccccccccc}\text { ODP } & 124 & 767 \mathrm{~B} & 5.9 & 4 & 3 & 20 & 3120 & 527.9 & \mathrm{~L} & \mathrm{Bio} / \mathrm{pmag} \\ \text { ODP } & 124 & 767 \mathrm{~B} & 5.9 & 4 & 3 & 90 & 3190 & 539.8 & \text { disperse } & \mathrm{Bio} / \mathrm{pmag} \\ \text { ODP } & 124 & 768 \mathrm{~B} & 10.5 & 7 & 4 & 107 & 5707 & 543.5 & \mathrm{~L} & \mathrm{Bio} / \mathrm{pmag} \\ \text { ODP } & 124 & 768 \mathrm{~B} & 10.5 & 7 & 5 & 129 & 5879 & 559.9 & \mathrm{~L} & \mathrm{Bio} / \mathrm{pmag} \\ \text { ODP } & 124 & 767 \mathrm{~B} & 5.9 & 4 & 4 & 134 & 3384 & 572.6 & \text { disperse } & \mathrm{Bio} / \mathrm{pmag} \\ \text { ODP } & 124 & 767 \mathrm{~B} & 5.9 & 5 & 1 & 17 & 3767 & 637.4 & \mathrm{~L} & \mathrm{Bio} / \mathrm{pmag} \\ \text { ODP } & 124 & 767 \mathrm{~B} & 5.9 & 5 & 1 & 22 & 3772 & 638.2 & \mathrm{~L} & \mathrm{Bio} / \mathrm{pmag} \\ \text { ODP } & 124 & 767 \mathrm{~B} & 5.9 & 5 & 1 & 40 & 3790 & 641.3 & \mathrm{~L} & \mathrm{Bio} / \mathrm{pmag} \\ \text { ODP } & 124 & 767 \mathrm{~B} / 769 \mathrm{~B} & 5.9 & 5 & 1 & 52 & 3802 & 643.3 & \mathrm{~L} & \mathrm{Bio} / \mathrm{pmag} \\ \text { ODP } & 124 & 767 \mathrm{~B} / 769 \mathrm{~B} & 5.9 & 5 & 3 & 21 & 4071 & 688.8 & \mathrm{~L} & \mathrm{Bio} / \mathrm{pmag} \\ \text { ODP } & 124 & 767 \mathrm{~B} / 769 \mathrm{~B} & 5.9 & 5 & 3 & 71 & 4121 & 697.3 & \mathrm{~L} & \mathrm{Bio} / \mathrm{pmag} \\ \text { ODP } & 124 & 767 \mathrm{~B} & 5.9 & 5 & 3 & 115 & 4165 & 704.7 & \mathrm{~L} & \mathrm{Bio} / \mathrm{pmag} \\ \text { ODP } & 124 & 767 \mathrm{~B} & 5.9 & 5 & 3 & 133 & 4183 & 707.8 & \mathrm{~L} & \mathrm{Bio} / \mathrm{pmag} \\ \text { ODP } & 124 & 767 \mathrm{~B} & 5.9 & 5 & 5 & 14 & 4364 & 738.4 & \mathrm{~L} & \mathrm{Bio} / \mathrm{pmag} \\ \text { ODP } & 124 & 767 \mathrm{~B} & 5.9 & 5 & 7 & 25 & 4675 & 791.0 & \mathrm{~L} & \mathrm{Bio} / \mathrm{pmag} \\ \text { ODP } & 124 & 767 \mathrm{~B} & 5.9 & 6 & 2 & 94 & 4944 & 836.5 & \mathrm{~L} & \mathrm{Bio} / \mathrm{pmag} \\ \text { ODP } & 124 & 767 \mathrm{~B} & 5.9 & 6 & 4 & 103 & 5253 & 888.8 & \mathrm{~L} & \mathrm{Bio} / \mathrm{pmag} \\ \text { ODP } & 124 & 767 \mathrm{~B} / 769 \mathrm{~B} & 5.9 & 6 & 4 & 115 & 5265 & 890.9 & \mathrm{~L} & \mathrm{Bio} / \mathrm{pmag} \\ \text { ODP } & 124 & 769 \mathrm{~B} / 768 \mathrm{~B} & 8.6 & 9 & 4 & 127 & 7767 & 903.1 & \mathrm{~L} & \mathrm{Bio} / \mathrm{pmag} \\ \text { ODP } & 124 & 769 \mathrm{~B} & 8.6 & 9 & 5 & 13 & 7803 & 907.3 & \mathrm{~L} & \mathrm{Bio} / \mathrm{pmag} \\ \text { ODP } & 124 & 768 \mathrm{~B} & 4.5 & 10 & 4 & 45 & 8495 & 935.4 & \mathrm{~L} & \mathrm{Bio} / \mathrm{pmag} \\ \text { ODP } & 124 & 769 \mathrm{~B} & 8.6 & 9 & 7 & 30 & 8120 & 944.2 & \mathrm{~L} & \mathrm{Bio} / \mathrm{pmag} \\ \text { ODP } & 124 & 769 \mathrm{~B} / 768 \mathrm{~B} & 8.6 & 10 & 1 & 43 & 8183 & 951.5 & \mathrm{~L} & \mathrm{Bio} / \mathrm{pmag} \\ \text { ODP } & 124 & 767 \mathrm{~B} & 5.9 & 7 & 1 & 124 & 5774 & 977.0 & \mathrm{~L} & \mathrm{Bio} / \mathrm{pmag} \\ \text { ODP } & 124 & 767 \mathrm{~B} & 5,90 & 7 & 4 & 76 & 6176 & 1045,01 & \mathrm{~L} & \mathrm{Bio} / \mathrm{pmag} \\ \text { ODP } & 124 & 7688 \mathrm{~B} & 4,50 & 11 & 2 & 129 & 9229 & 1098,52 & \mathrm{~L} & \mathrm{Bio} / \mathrm{pmag} \\ \text { ODP } & 125 & 7688 \mathrm{~B} & 4,50 & 11 & 4 & 85 & 9485 & 1155,41 & \mathrm{~L} & \mathrm{Bio} / \mathrm{pmag} \\ & & & & & & & & & & \end{array}$

\section{$2 \quad$ Dating Errors}

Ages for the ash layers are determined using various approaches. Ages for on-land tephras are based on ${ }^{14} \mathrm{C}$ and ${ }^{40} \mathrm{Ar} /{ }^{39} \mathrm{Ar}$ dating and are generally more precise than ages for marine tephras. Ages for many marine tephras, such as those found in CAVA gravity cores (Kutterolf et al., 2008) and New Zealand gravity cores (Lowe et al., 2008), are determined through correlation to radiometrically dated on-land tephras. Finally, ages are estimated from sedimentation rates for tephras found in DSDP, ODP, and IODP sites along the Ring of Fire. Table DR2 lists the age of tephras used in this study, an estimate of the error, and the dating method used.

Age dating of tephra layers involves both random and systematic errors. Precise estimates of these errors across the entire record are difficult to establish due to the composite nature of the data set and differences in the applied dating methods. Our estimates of the total error are summarized in Table DR3. 
The dominant random error for nearly all samples is associated with the depth estimation of relevant time markers in cores, either of the tephras themselves, biostratigraphic markers or the Brunhes-Matayama boundary. The error in depth estimation varies depending on location and borehole conditions. These errors propagate into the estimates of sedimentation rates that are used, especially for the deep sea drilling cores, to date the tephras. Additionally, the time markers themselves are subject to dating errors.

To estimate an error for ages determined using sedimentation rates, we made use of tephras from CAVA IODP/ODP cores where the sampled material allowed compositional analyses using an electron microprobe. Specifically, glass and mineral compositions (major and minor elements) were analysed for at least 15 individual glass shards (per tephra layer) with a JEOL JXA 8200 wavelength dispersive electron microprobe (EMP) at GEOMAR in Kiel (for a detailed discussion of analytical methods see Kutterolf et al., 2011). Using these measurements, we were able to preliminarily correlate 10 ash layers across several sites with major element data, which allowed a cross-check of different age models. The difference in ages determined using distinct age models yields a relatively small error estimate for the age inferred from sedimentation rates. In addition, we have also been able to interrogate the age models by correlating, compositionally, 5 younger tephra layers (up to $191 \mathrm{ka}$ ) to well-dated $\left({ }^{14} \mathrm{C},{ }^{40} \mathrm{Ar} /{ }^{39} \mathrm{Ar}\right.$ ) eruptions from field samples (e.g. Kutterolf et al 2008; see data table). The error estimates obtained from cross-correlation of marine cores (less than $2 \%$ for tephras older than $300 \mathrm{ka}$, or 1-10 kyrs) or correlation to dated onland tephras (up to $6 \%$, or 1-4 kyrs) are relatively small for the CAVA sites during the first 400 kyr. We assume that the level of random error for all ROF data is comparable to the error we determined from our detailed analysis of this subset.

Superimposed on the random error are systematic errors. Re-mineralization of magnetic minerals leads to an underestimation of sedimentation rates with increasing time, when these rates are based on paleomagnetics, and a bias in tephras ages toward later times (Channel and Stoner 2002). A further bias in this direction is associated with tephras in regions characterized by sedimentation rates of less than $5 \mathrm{~cm} / \mathrm{kyr}$ (Guyodo and Channell, 2002), which applies to approximately $30 \%$ of the data set (see Table DR 1 ). Another systematic bias may be associated with missing tephras layers due to: a) non-recovery of the upper few meters of cores (relevant for recent tephras but not for gravity core tephras from New Zealand and Central America); b) destructive drilling techniques (relevant for older cores); and c) the lower number of deeper old tephras identified within the core.

An opposite bias of $6.4 \%$ would be associated with correcting the Brunhes-Matayama boundary age of $730 \mathrm{ka}$, adopted for some older cores, to the current estimate of $710 \mathrm{ka}$. However, we have only traced the adoption of the former value to two locations (Tonga and Philippines). 
Table DR2: Table of correlations established between different DSDP, ODP and IODP sites and with dated on-land tephras at CAVA sites that were used to constrain age models.

\begin{tabular}{|c|c|c|c|c|c|c|c|c|c|c|c|c|c|c|c|c|c|c|c|c|c|}
\hline Leg/Exp & Site & Hole & Core & Type & Section & Top & Bottom & $\begin{array}{c}\text { depth } \\
{[\mathrm{cm}]}\end{array}$ & $\begin{array}{l}\text { sedrate } \\
{[\mathrm{cm} / \mathrm{ka}]}\end{array}$ & age $[\mathrm{ka}]$ & $\begin{array}{l}\text { deviation } \\
\text { age (ka) }\end{array}$ & $\begin{array}{c}\text { error in } \\
\% \\
\end{array}$ & $\mathrm{Na}_{2} \mathrm{O}$ & $\mathrm{K}_{2} \mathrm{O}$ & $\mathrm{FeO}_{\mathrm{t}}$ & $\mathrm{SiO}_{2}$ & $\mathrm{CaO}$ & MgO & $\mathrm{Al}_{2} \mathrm{O}_{3}$ & $\mathrm{TiO}_{2}$ & Alkalis \\
\hline \multicolumn{22}{|c|}{ Correlations to well-dated field tephras } \\
\hline PAT & & & & & & & & & & 23 & \pm 2 & & 4,12 & 4,15 & 0,69 & 77,19 & 0,43 & 0,05 & 13,17 & 0,08 & 8,26 \\
\hline \multicolumn{14}{|r|}{0,16} & 0,16 & 0,09 & 0,36 & 0,04 & 0,02 & 0,13 & 0,08 & 0,24 \\
\hline 138 & 844 & A & 1 & $\mathrm{H}$ & 1 & 19 & 24 & 24 & 1,11 & 22 & -1 & $-6,0$ & 3,70 & 3,96 & 0,55 & 77,87 & 0,70 & 0,09 & 12,95 & 0,09 & 7,66 \\
\hline LAT & & & & & & & & & & 25 & $\pm 0,45$ & & 3,87 & 2,87 & 1,73 & 75,94 & 1,66 & 0,33 & 13,72 & 0,30 & 6,65 \\
\hline \multicolumn{14}{|r|}{0,48} & 0,10 & 0,14 & 0,69 & 0,12 & 0,07 & 0,35 & 0,12 & 0,50 \\
\hline 84 & 569 & & 1 & $\mathrm{H}$ & 1 & 72 & 80 & 80 & 3,3 & 24 & -1 & $-2,2$ & 4,09 & 3,07 & 1,67 & 75,19 & 1,60 & 0,34 & 13,66 & 0,23 & 7,16 \\
\hline Los Cocoyos & & & & & & & & & & 84 & \pm 2 & & 3,39 & 4,02 & 0,51 & 77,93 & 0,50 & 0,09 & 12,89 & 0,07 & 7,49 \\
\hline \multicolumn{14}{|r|}{0,26} & 0,07 & 0,07 & 0,28 & 0,06 & 0,02 & 0,15 & 0,08 & 0,22 \\
\hline 66 & 496 & & 5 & $\mathrm{H}$ & 5 & 130 & 1350 & 230 & $100 / 14$ & 81 & -3 & $-3,6$ & 3,70 & 4,12 & 0,77 & 77,63 & 0,50 & 0,06 & 13,20 & 0,07 & 7,84 \\
\hline 66 & 487 & & 3 & $\mathrm{H}$ & 3 & 90 & 98 & 1450 & 17,6 & 82 & -2 & $-1,9$ & 3,35 & 3,92 & 0,53 & 78,36 & 0,63 & 0,09 & 12,95 & 0,08 & 7,28 \\
\hline 66 & 487 & & 3 & $\mathrm{H}$ & 4 & 40 & 51 & 1553 & 17,6 & 88 & 4 & 5,0 & 3,43 & 3,84 & 0,52 & 78,36 & 0,65 & 0,09 & 12,93 & 0,09 & 7,27 \\
\hline WFT & & & & & & & & & & 159 & \pm 3 & & 3,20 & 4,41 & 0,62 & 77,51 & 0,61 & 0,07 & 12,83 & 0,06 & 7,60 \\
\hline \multicolumn{14}{|r|}{0,14} & 0,24 & 0,07 & 0,20 & 0,03 & 0,02 & 0,13 & 0,08 & 0,20 \\
\hline 67 & 496 & & 6 & $\mathrm{H}$ & $\mathrm{CC}$ & 10 & 65 & 5310 & $100 / 14$ & 158 & -1 & $-0,5$ & 4,12 & 4,06 & 0,70 & 77,20 & 0,40 & 0,06 & 13,27 & 0,08 & 8,18 \\
\hline LFT & & & & & & & & & & 191 & \pm 11 & & 3,97 & 4,30 & 1,01 & 75,93 & 0,78 & 0,15 & 13,51 & 0,14 & 8,27 \\
\hline \multicolumn{14}{|r|}{0,09} & 0,15 & 0,13 & 0,25 & 0,03 & 0,02 & 0,12 & 0,05 & 0,19 \\
\hline 67 & 496 & & 7 & $\mathrm{H}$ & 2 & 120 & 125 & 5720 & $100 / 14$ & 188 & -3 & $-1,6$ & 4,12 & 4,26 & 1,00 & 76,20 & 0,70 & 0,16 & 13,27 & 0,18 & 8,28 \\
\hline
\end{tabular}

Correlations to other ODP cores in

\begin{tabular}{|c|c|c|c|c|c|c|c|c|c|c|c|c|c|c|c|c|c|c|c|c|c|}
\hline $\begin{array}{l}\text { Correlations to other } \\
\text { icinity }\end{array}$ & & & & & & & & & & & & & & & & & & & & & \\
\hline 67 & 499 & & 2 & $\mathrm{H}$ & 5 & 130 & 135 & 830 & 100 & 8 & -12 & $-59,8$ & 3,64 & 3,59 & 0,94 & 77,55 & 0,99 & \begin{tabular}{|l|l}
0,14 \\
\end{tabular} & \begin{tabular}{|l}
12,92 \\
\end{tabular} & 0,13 & 7,24 \\
\hline 67 & 498 & & 1 & $\mathrm{H}$ & 3 & 25 & 30 & 330 & 10 & 33 & 12 & 59,8 & 3,74 & 3,52 & 0,98 & 77,48 & 0,97 & 0,15 & $\begin{array}{l}12,92 \\
\end{array}$ & 0,13 & 7,27 \\
\hline 138 & 844 & B & 1 & $\mathrm{H}$ & 1 & 115 & 120 & 120 & 1,11 & 108 & -5 & $-4,4$ & 3,69 & \begin{tabular}{|l}
4,04 \\
\end{tabular} & 0,53 & 78,20 & 0,64 & \begin{tabular}{|l}
0,08 \\
\end{tabular} & \begin{tabular}{|l}
12,67 \\
\end{tabular} & 0,09 & 7,73 \\
\hline 202 & 1241 & A & 1 & $\mathrm{H}$ & 2 & 9 & 14 & 164 & 1,39 & 118 & 5 & 4,4 & 3,71 & 4,00 & 0,53 & 77,98 & 0,62 & \begin{tabular}{|l} 
\\
\end{tabular} & \begin{tabular}{|l}
12,92 \\
\end{tabular} & 0,08 & 7,71 \\
\hline 138 & 844 & A & 1 & $\mathrm{H}$ & 3 & 0 & 30 & 330 & 1,11 & 297 & -6 & $-1,9$ & 3,90 & \begin{tabular}{|l}
3,89 \\
\end{tabular} & 0,54 & 78,09 & 0,62 & \begin{tabular}{|l}
0,08 \\
\end{tabular} & 12,69 & 0,09 & 7,79 \\
\hline 66 & 487 & & 7 & $\mathrm{H}$ & 4 & 25 & 35 & 5435 & 17,6 & 309 & 6 & 1,9 & 3,84 & \begin{tabular}{|l}
3,88 \\
\end{tabular} & 0,48 & 77,76 & 0,70 & 0,09 & 13,09 & 0,07 & $\begin{array}{r}7,22 \\
\end{array}$ \\
\hline 67 & 499 & & 14 & $\mathrm{H}$ & 2 & 30 & 42 & 11692 & $100 / 30$ & 365 & -17 & -5 & 3,52 & \begin{tabular}{|l}
4,19 \\
\end{tabular} & 0,59 & 77,89 & 0,63 & 0,09 & $\mid 12,93$ & 0,09 & 7,71 \\
\hline 67 & 494 & A & 1 & $\mathrm{H}$ & 2 & 70 & 97 & 3997 & 10 & 400 & -53 & -12 & 3,34 & 3,94 & 0,56 & 77,68 & 0,88 & 0,11 & 13,13 & 0,18 & 7,28 \\
\hline 202 & 1242 & A & 6 & $\mathrm{H}$ & 6 & 54 & 59 & 5279 & 12 & 507 & -5 & $-1,1$ & 4,43 & 4,44 & 3,92 & 66,70 & 2,52 & 0,93 & 15,86 & 0,88 & 8,87 \\
\hline 202 & 1242 & D & 5 & $\mathrm{H}$ & 1 & 50 & 60 & 5410 & 12 & 518 & 5 & 1,1 & 4,83 & 4,32 & 3,99 & 66,35 & 2,66 & 1,03 & 15,60 & 0,87 & 9,15 \\
\hline
\end{tabular}




\begin{tabular}{|c|c|c|c|c|c|c|c|c|c|c|c|c|c|c|c|c|c|c|c|c|c|}
\hline 202 & 1242 & A & 8 & $\mathrm{H}$ & 1 & 101 & 105 & 6475 & 12 & 606 & -10 & $-1,7$ & $\begin{array}{l}3,32 \\
\end{array}$ & 3,85 & 0,99 & 77,14 & 1,38 & 0,31 & 12,84 & 0,12 & 7,17 \\
\hline 202 & 1242 & $D$ & 6 & $\mathrm{H}$ & 3 & 120 & 125 & 6725 & 12 & 627 & 10 & 1,7 & 3,18 & 3,84 & 0,96 & 76,94 & 1,47 & 0,20 & 13,23 & 0,13 & 7,01 \\
\hline 202 & 1242 & C & 8 & $\mathrm{H}$ & 4 & 7 & 10 & 7210 & 12 & 668 & 13 & 1,9 & 3,61 & 3,49 & 1,31 & 75,25 & 1,61 & 0,34 & 14,01 & 0,28 & 7,10 \\
\hline 67 & 499 & & 22 & $\mathrm{H}$ & 6 & 128 & 135 & 19985 & $100 / 30$ & 642 & -13 & $-1,9$ & 3,50 & 3,79 & 1,57 & 75,24 & 1,24 & 0,26 & 14,03 & 0,25 & 7,29 \\
\hline 202 & 1242 & C & 11 & $\mathrm{H}$ & 3 & 119 & 132 & 10032 & 12 & 903 & -5 & $-0,5$ & 4,03 & 5,51 & 2,05 & 69,30 & 1,40 & 0,57 & 16,31 & 0,63 & 9,54 \\
\hline 202 & 1242 & A & 11 & $\mathrm{H}$ & 7 & 6 & 20 & 10140 & 12 & 912 & 5 & 0,5 & 4,55 & 5,69 & 2,25 & 68,60 & 1,32 & 0,53 & 16,25 & 0,62 & 10,24 \\
\hline 67 & 495 & & 5 & $\mathrm{H}$ & 4 & 5 & 15 & 4260 & 3,7 & 1151 & -8 & $-0,7$ & 3,85 & 3,52 & 1,42 & 76,10 & 1,24 & 0,22 & 13,38 & 0,19 & 7,36 \\
\hline 202 & 1242 & A & 15 & $\mathrm{H}$ & 2 & 48 & 49 & 13219 & 12 & 1168 & 8 & 0,7 & 3,57 & 3,85 & 1,31 & 76,35 & 1,23 & 0,18 & 13,34 & 0,10 & 7,43 \\
\hline 67 & 497 & & 37 & $\mathrm{H}$ & 6 & 70 & 85 & 34785 & $90 / 3,22$ & 4439 & 17 & 0,4 & 3,63 & 3,88 & 1,04 & 76,89 & 0,92 & 0,17 & 13,21 & 0,16 & 7,50 \\
\hline 138 & 844 & B & 4 & $\mathrm{H}$ & 4 & 92 & 102 & 2902 & 0,67 & 4405 & -17 & $-0,4$ & 3,79 & 4,28 & 0,88 & 77,15 & 0,85 & 0,10 & 12,76 & 0,12 & 8,07 \\
\hline
\end{tabular}


Table DR3: Error evaluation for specific ODP/IODP sites around the ROF

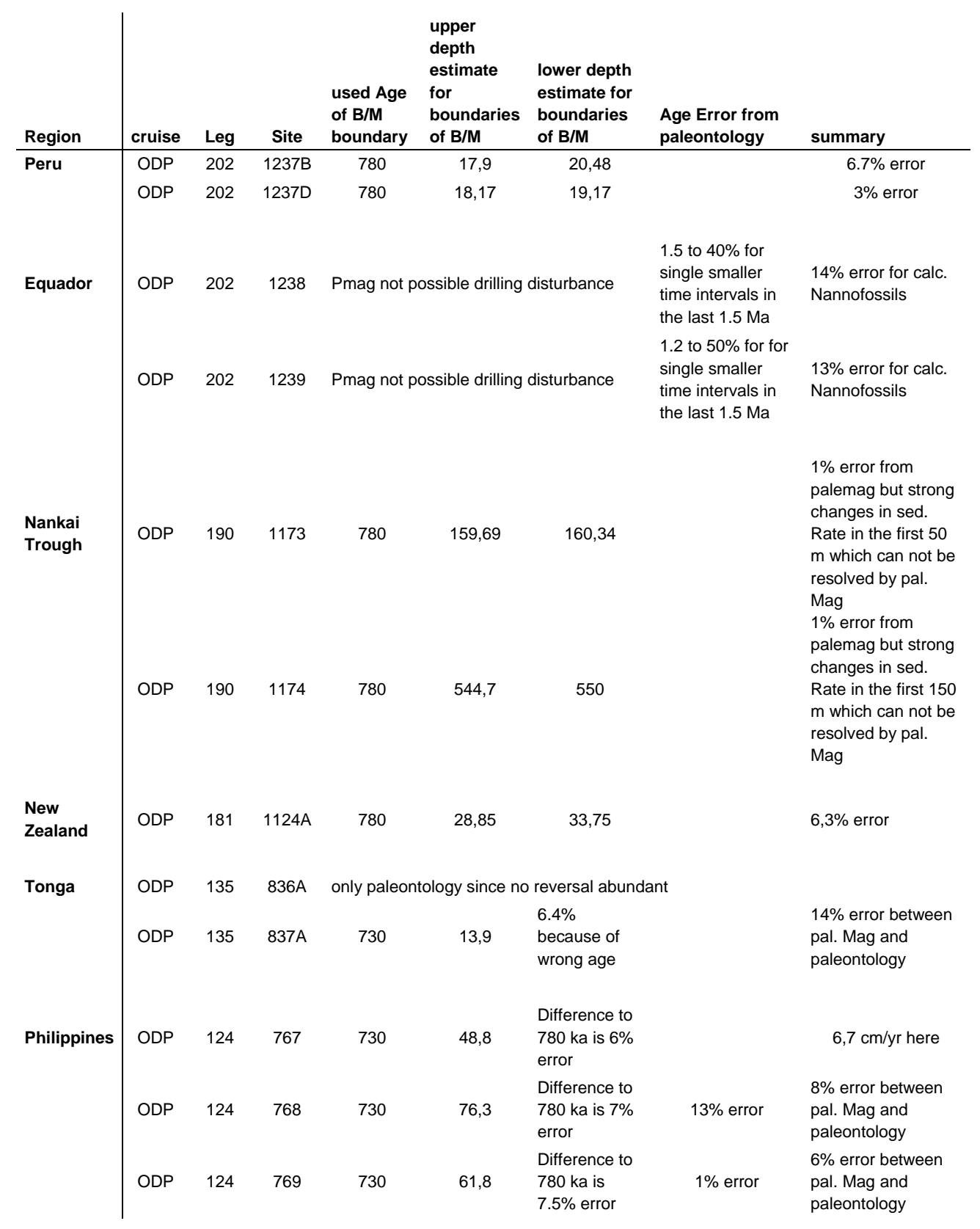




\section{Statistical Analyses}

Evaluation of the spectral content of the tephra time-series is performed using a multitaper procedure (Percival and Walden, 1998; Thomson, 1982) with a time bandwidth (tbw) of 1.5. The background continuum is estimated by fitting an exponential function of frequency to the spectral power estimate between frequencies of $1 / 80 \mathrm{kyr}$ and $1 / 10 \mathrm{kyr}$, and the peak that extends furthest above the background continuum, in a fractional sense, within $14 \%$ of the $1 / 41 \mathrm{kyr}$ obliquity frequency is identified. This peak is at $1 / 44 \mathrm{kyr}$ and rises 6.3 times above the background continuum (Fig. DR1).

We use a Monte Carlo procedure to evaluate the statistical significance of this peak, taking into account the fact that time uncertainty leads us to broaden the search away from precisely at the obliquity frequency. To realize synthetic time series of tephra layers consistent with a null hypothesis of episodic eruptions with no periodic component, we draw values from a Poisson distribution at 500 years steps, with a probability of $\lambda=0.25$ that an event will occur within each step. Time-series are 1200 kyrs long, giving 600 events on average. A single realization of such a time series is shown in Figure DR2 along with its spectral estimate. Spectral estimates are obtained and the largest peak within $14 \%$ of $1 / 41 \mathrm{kyr}$ is recorded as described in the foregoing paragraph. Repeating this procedure 100,000 times allows for building up an approximate distribution for the null hypothesis (Fig. DR2).

We find that realizations have a ratio relative to the estimated background continuum that equal or exceed the observed value of 6.3 less than $1 \%$ of the time. More exactly, the fraction of realizations greater than 6.3 is $0.48 \%$. These results indicate that the peak is statistically significant. We are aware of no other viable process that would concentrate spectral energy in this frequency band and, thus, conclude that there is a significant response to changes in Earth's obliquity.

For purposes of comparison, we also show in Figure DR3 the time-series and spectral estimates from the tephra time series in relation to those associated with orbital variations (Berger and Loutre, 1992) and the rate-of-change of benthic $\delta^{18} \mathrm{O}$ (Lisiecki and Raymo, 2005). In this case spectral estimates are shown on a linear axis. For the tephra time series both the compressed and uncompressed version are shown. Reducing the age of all points by $10 \%$ leads to $10 \%$ shift toward higher frequencies in the associated spectral estimate.

Figure DR4 shows a series of spectral estimates from sequentially older $400 \mathrm{kyr}$ segments of the tephra time series. All segments show some concentration of spectral energy at frequencies within $14 \%$ of the $1 / 41 \mathrm{kyr}$ obliquity band., particularly within the last $800 \mathrm{kry}$. Furthermore, the peak frequency for the most recent $400 \mathrm{kyr}$ is centered at $1 / 41 \mathrm{kyr}$ but decreases to $1 / 46 \mathrm{kyr}$ for the oldest time window. We attribute the general decrease in frequency and amplitude with age to increased timing error and reduced sampling coverage, with a relatively smaller number of events identified in older sediments. These effects introduce non-stationarity in the record and are presumed to cause the peak near obliquity to 
appear at $1 / 44$ kyr when the entirety of the record is analyzed. We partially correct for this bias by compressing the time series by $10 \%$. This compression yields no age change most recently, a $6 \mathrm{kyr}$ decrease in age at $600 \mathrm{kyr}$, etc. These shifts are within the estimated error range of the dating and are meant to adjust for the plausible systematic errors in the chronology, though more detailed timing errors doubtlessly still exist.

Figure DR1: Multi-tape power spectral estimate of the $1200 \mathrm{kyr}$ long tephra time series using a time-bandwidth product of 1.5 . Vertical dashed lines are at frequencies of $1 / 36 \mathrm{kyr}$ and 1/48 kyr, i.e. $1 / 41 \mathrm{kry}+/-14 \%$, and show the frequency interval where the maximum peak is selected from. Diagonal dashed line is an exponential fit to the background continuum between frequencies of 1/10 kyr and 1/80 kyr plotted on logarithmic axes. The observed peak at $1 / 44 \mathrm{kyr}$ rises a factor of 6.3 above the fit to the background continuum.

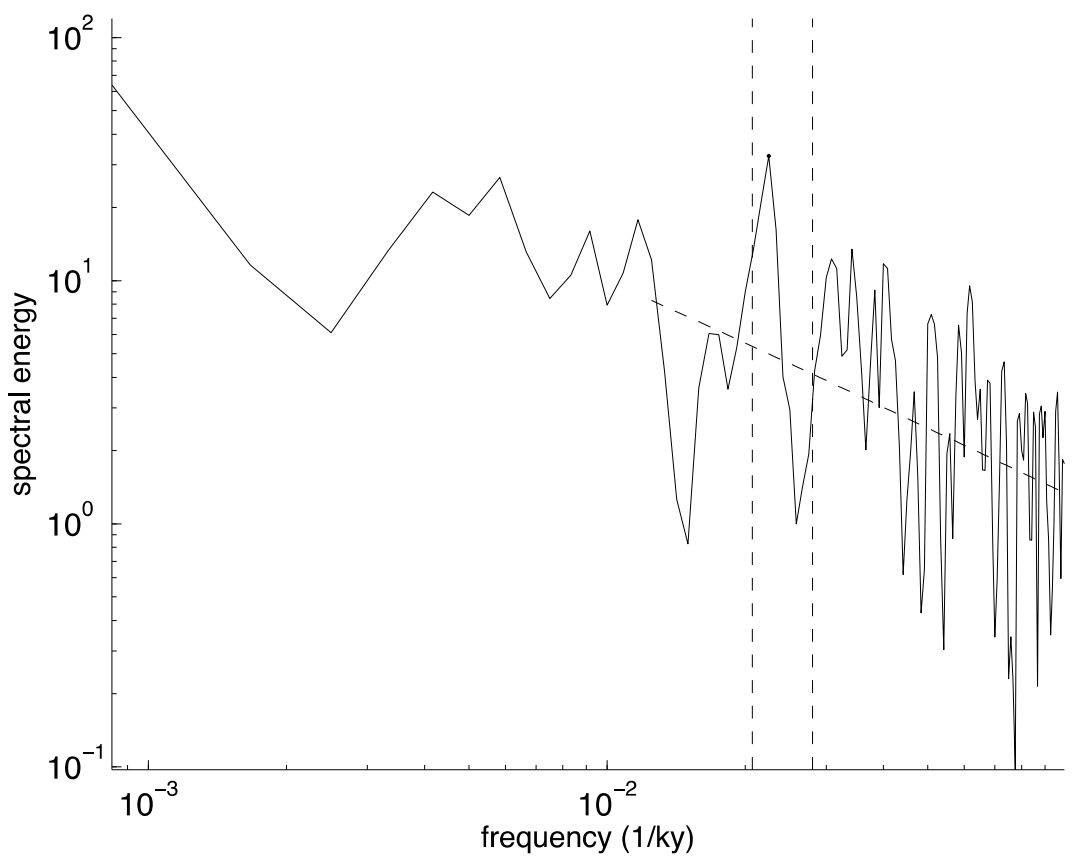


Figure DR2: Monte Carlo test of significance. (Top) A synthetic tephra time series running from -1200 kyr to the present. (Middle) The spectral estimate derived from the synthetic time series, with the maximum peak relative to the background continuum within $14 \%$ of $1 / 41 \mathrm{kyr}$ identified. This peak rises 3.0 times the background continuum. Note that axes are logarithmic. (Bottom) A histogram of the null distribution estimated by identifying peaks analogous to that represented in the above two panels 100,000 times. The vertical dashed line indicates the observed ratio of 6.3 . Less than $1 \%$ of all realization lie to the right of this observed value.
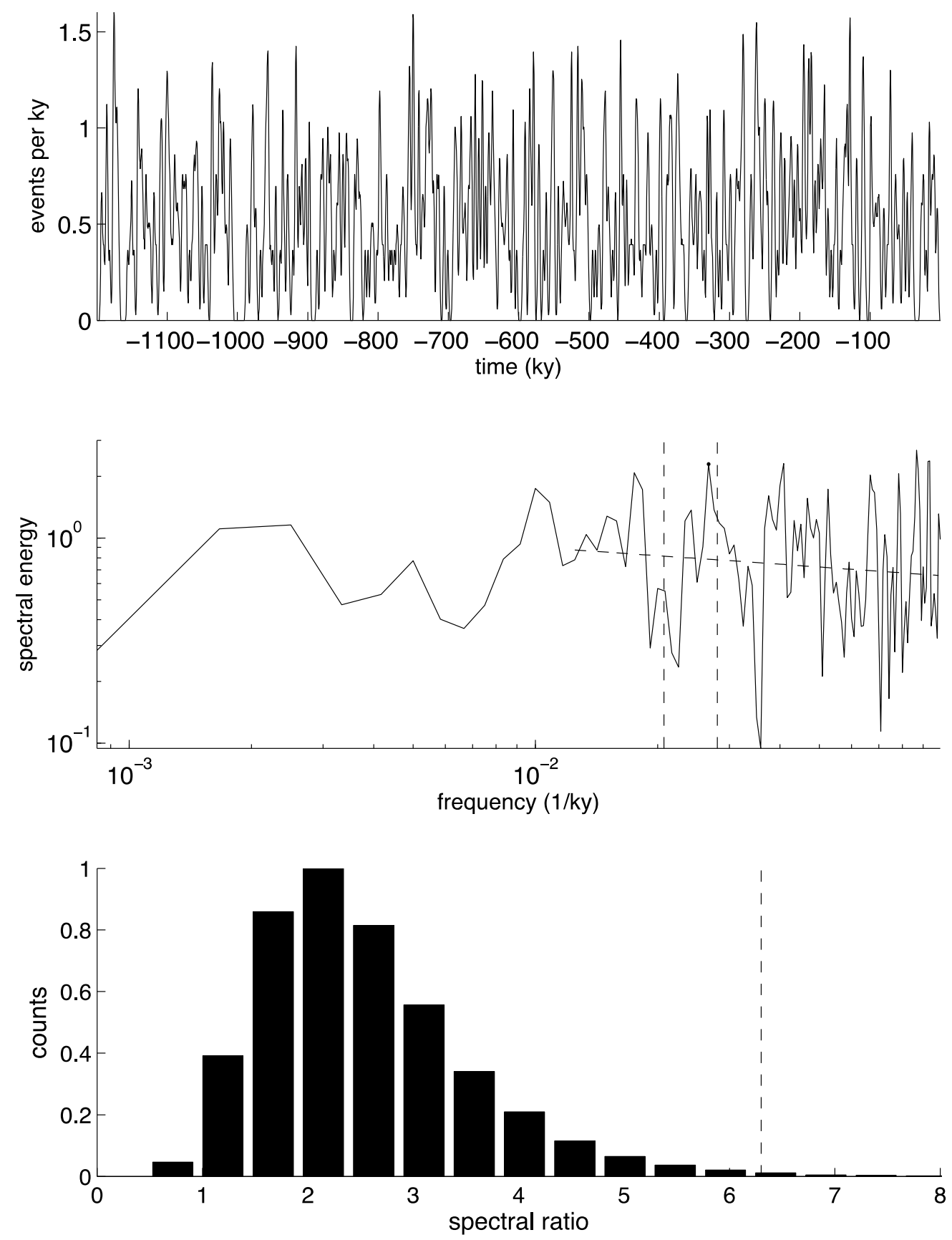
Figure DR3: (A) Compressed (solid black line) and uncompressed (dashed red line) ROF ash time series filtered with a stable phase running average low pass filter (window length $5 \mathrm{kyr}$ ) (B) Time series of $\delta^{18} \mathrm{O}$ (Lisiecki and Raymo, 2005) (C) Time derivative of the $\delta^{18} \mathrm{O}$ time series in frame B. (D) Power spectra of the compressed (solid black line) and uncompressed (dashed red line) ash time series (frame A) calculated with a multi-taper spectral analysis using a time band width of 1.5. In contrast to Fig. $1 \mathrm{~B}$ in the main text, the power spectrum in frame D does not show the $95 \%$ confidence interval and is not normalized using the peak in the obliquity band. (E) Power spectrum of orbital variability, a composite time series composed of equal parts variability from obliquity and precession. (F) Power spectra of the time derivative of $\delta^{18} \mathrm{O}$ records (Lisiecki and Raymo, 2005) (frame C)
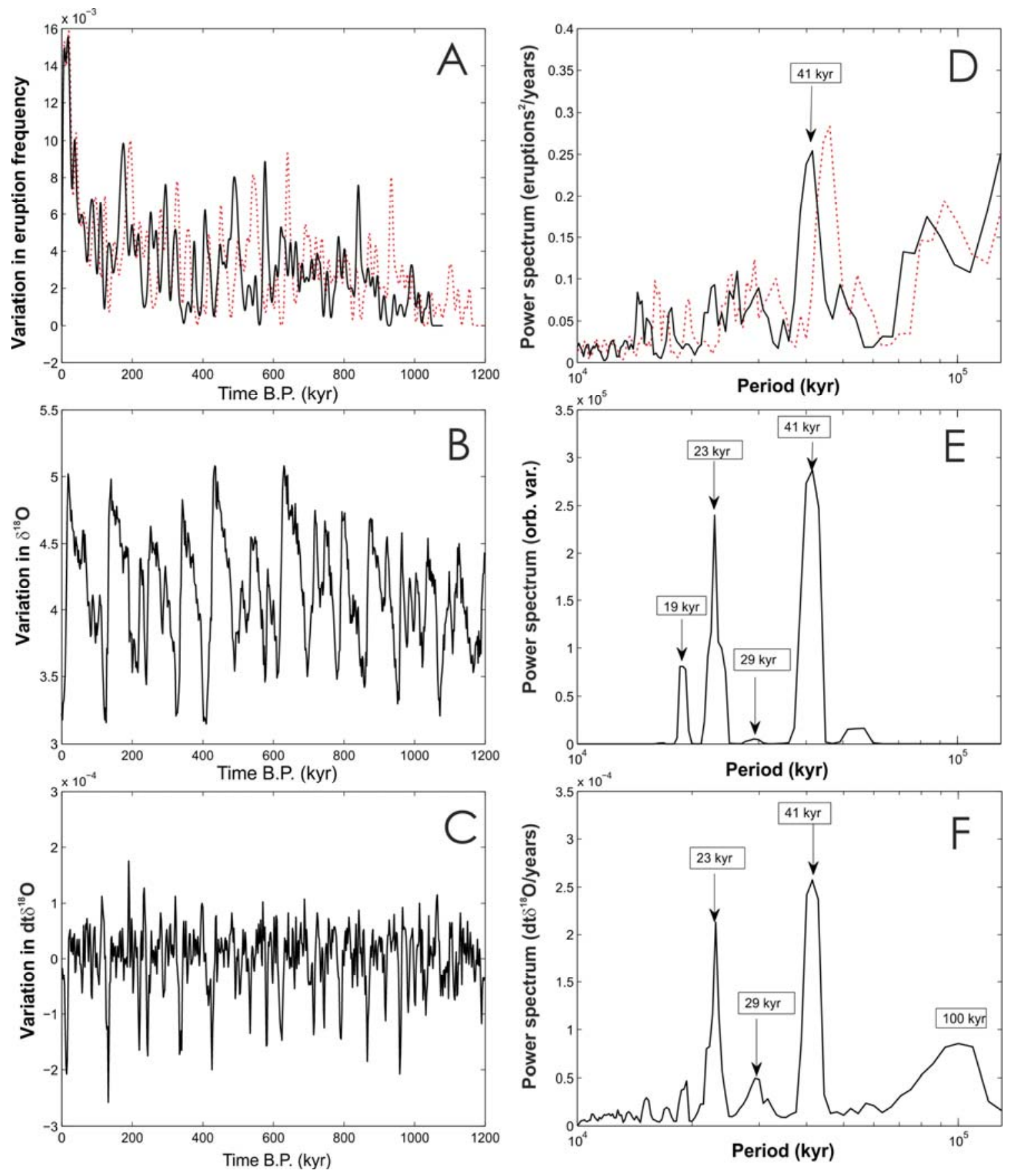
Figure DR4: Power spectral estimates of the ash time series. Individual spectral are estimated for time windows of widths $400 \mathrm{kyr}$ at ages centered at $200 \mathrm{kyr}, 400 \mathrm{kyr}, 600 \mathrm{kyr}$ and $800 \mathrm{kyr}$. In this case, the multi-taper bandwidth is 2 . Black line denotes the power spectra obtained using the entire record. The data subsets up to $800 \mathrm{kyr}$ show a relatively consistent spectral structure near the obliquity band (blue vertical bar), but a decrease in peak frequency and amplitude at greater ages. The dashed vertical lines indicate the precession bands near $1 / 19 \mathrm{kyr}$ and $1 / 23 \mathrm{kry}$, the vertical peach bar is at $1 / 26 \mathrm{kyr}$, and the light gray bar is at $1 / 100$ kyr.

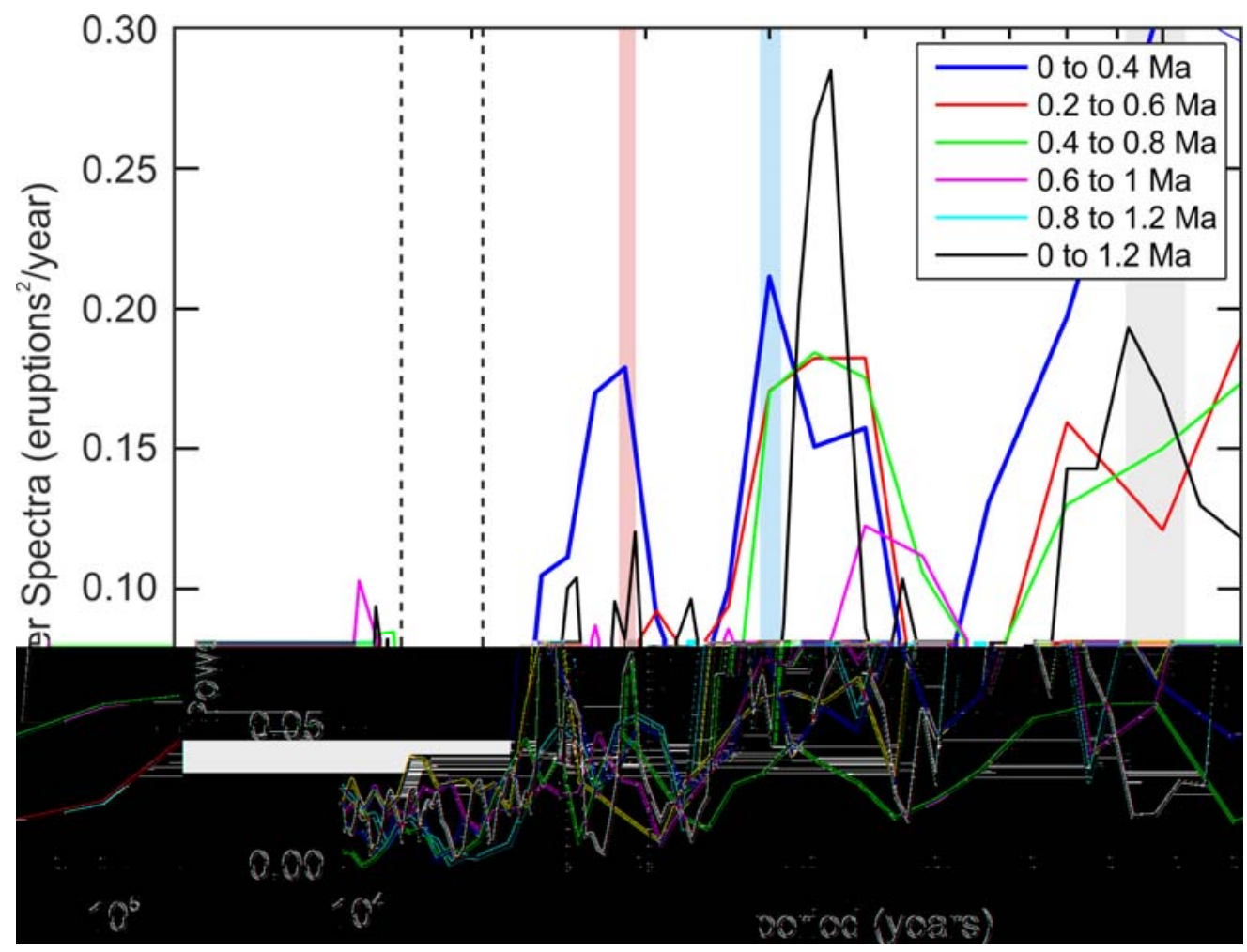




\section{Stress Calculations}

To compute ice-age induced crustal stresses, we adopt a standard normal mode methodology valid for spherically symmetric, self-gravitating, Maxwell viscoelastic Earth models (Peltier, 1974; Wu and Peltier, 1982). In particular, this methodology yields depth dependent radial and tangential displacements and stress, and the gravitational potential perturbation, arising from an impulse forcing (Wu and Peltier, 1982; Eqs. 5,7). The response to an arbitrary surface mass loading is computed by convolving this impulse response, in both space and time, with the surface mass load (ice plus ocean) load (Mitrovica et al., 1994).

Here, we have chosen to highlight the geometry and time history of the radial stress (Wu and Peltier, 1982; Eq. 7) at the specific depth of $20 \mathrm{~km}$ (see text). This depth is chosen because it is a characteristic depth associated with magma chambers that feed surface volcanism.

The Earth model we adopt in this study has a density and elastic structure given by the seismic model PREM (Dziewonski and Anderson, 1981). In addition, we use the coupled ice history-radial viscosity model ICE5G-VM2 derived by Peltier (2004). ICE-5G provides the space-time history of ice cover over the last glacial cycle of the ice age. VM2 is a radial profile of sub-lithospheric mantle viscosity that is characterized by values of $\sim 5 \times 10^{20} \mathrm{~Pa} \mathrm{~s}$ in the upper mantle and $2-3 \times 10^{21} \mathrm{~Pa}$ in the lower mantle, and an elastic lithosphere of thickness $90 \mathrm{~km}$. Finally, the ocean load component of the total surface mass load is computed using a gravitationally self-consistent sea-level theory that accounts for the migration of shorelines, changes in grounded, marine-based ice, and the feedback into sea level of contemporaneous perturbations in the Earth's rotation vector (Kendall et al., 2005). 
Figure DR5. Numerical prediction of the ice age induced rate of change of radial stress at 20 $\mathrm{km}$ depth (units of $\mathrm{MPa} / 10 \mathrm{kyr}$ ) at $10 \mathrm{ka}$ (i.e., during the final deglaciation phase). The inset $\mathrm{B}$ is a detail of the global plot in frame $\mathrm{A}$ in the vicinity of Central America, which is also shown in Figure 4 of the main manuscript.

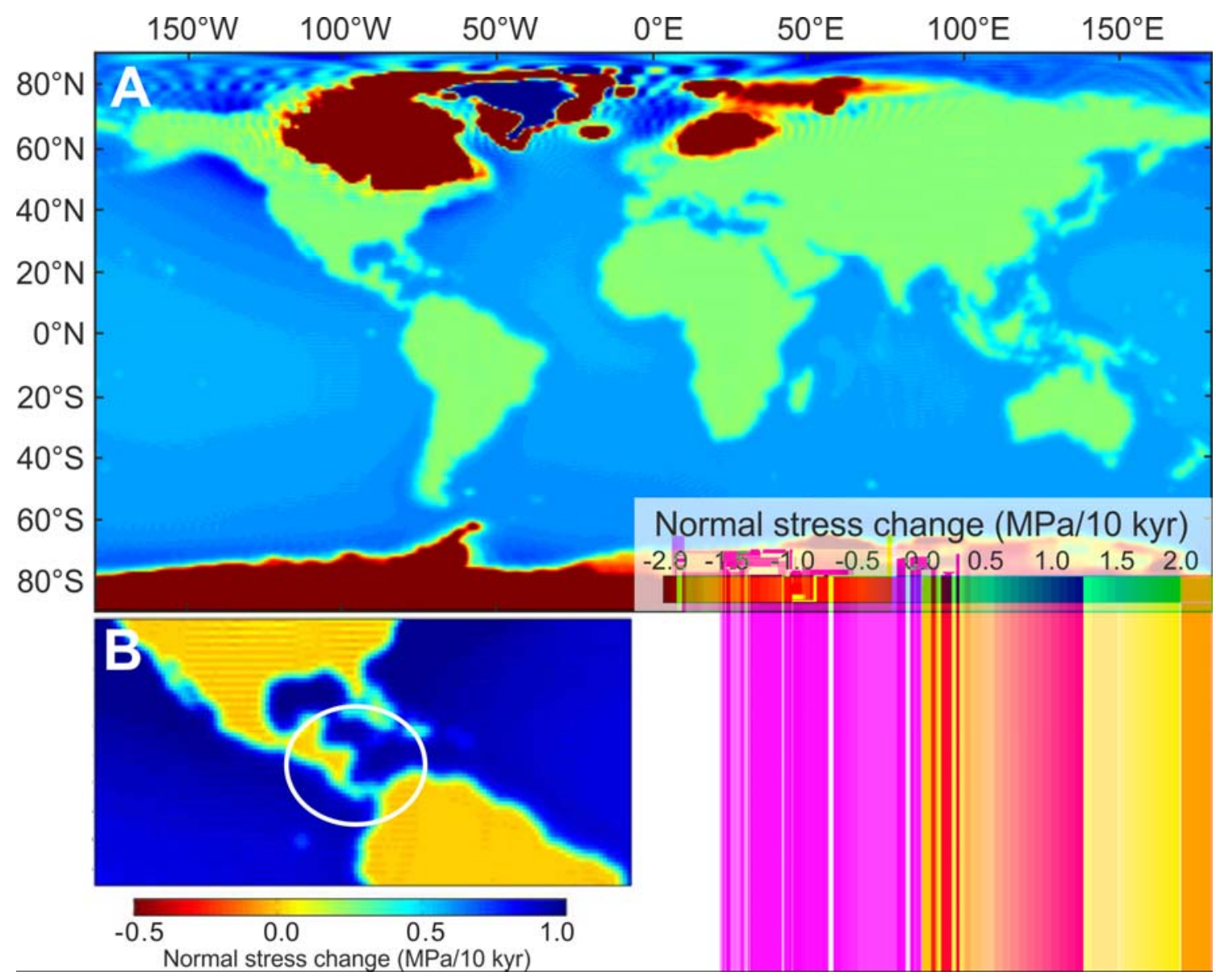




\section{$5 \quad$ References}

Aubouin, J., von Huene, R., and al., e., 1982a, Site 496: Middle America Trench Upper Slope, in Office, U. S. G. P., ed., Init. Repts. DSDP, Volume 67/Site 496: Washington.

Aubouin, J., von Huene, R., and al., e., 1982b, Site 497: Middle America Trench Upper Slope, in Office, U. S. G. P., ed., Init. Repts. DSDP, Volume 67/Site 497: Washington.

Aubouin, J., von Huene, R., and al., e., 1982c, Site 499: Middle America Trench Upper Axis, in Office, U. S. G. P., ed., Init. Repts. DSDP, Volume 67/Site 499: Washington.

Bowles, F. A., Jack, R. N., and Carmichael, I. S. E., 1973, Investigation of deep-sea volcanic ash layers from equatorial Pacific cores: Geol. Soc. Am. Bull., v. 84, p. 2371-2388.

Clift, P. D., Chan, L. H., Blusztajn, J., Layne, G. D., Kastner, M., and Kelly, R. K., 2005, Pulsed subduction accretion and tectonic erosion reconstructed since $2.5 \mathrm{Ma}$ from the tephra record offshore Costa Rica.: Geochem. Geophys. Geosys., v. 6, no. 9, p. 1-21.

Creager, J. S., and Scholl, D. W., 1973a, Site 183, in Office, U. S. G. P., ed., Init. Repts. DSDP, Volume 19/ Site 183: Washington.

Creager, J. S., and Scholl, D. W., 1973b, Site 190, in Office, U. S. G. P., ed., Init. Repts. DSDP, Volume 19/ Site 190: Washington.

Creager, J. S., and Scholl, D. W., 1973c, Site 192.

Dziewonski, A.M., and Anderson, D.L., 1981, Preliminary reference Earth model (PREM): Phys. Earth Planet. Int., v. 25, p. 297-356.

Kendall, R.A., Mitrovica, J.X., and Milne, G.A., 2005, On post-glacial sea level II. Numerical formulation and comparative results on spherically symmetric models: Geophys. J. Int., v. 161, p. 679-706.

Kimura, G., Silver, E. A., and Blum, P., 1997, Costa Roca accretionary wedge.

Kulm, L. D., and von Huene, R., 1973a, Site 178, in Office, U. S. G. P., ed., Init. Repts. DSDP, Volume 18/ Site 178: Washington.

Kulm, L. D., and von Huene, R., 1973b, Site 179, in Office, U. S. G. P., ed., Init. Repts. DSDP, Volume 18/ Site 179: Washington.

Kutterolf, S., Freundt, A., and Burkert, C., 2011, Eruptive history and magmatic evolution of the 1.9 kyr Plinian dacitic Chiltepe Tephra from Apoyeque volcano in west-central Nicaragua: Bull. Volcanol., v. 73, p. 811-831.

Kutterolf, S., Freundt, A., Peréz, W., Mörz, T., Schacht, U., Wehrmann, H., and Schmincke, H.U., 2008, The Pacific offshore record of Plinian arc volcanism in Central America, part 1: Along-arc correlations: Geochem. Geophys. Geosyst., v. 9, no. 2, p. doi:10.1029/2007GC001631.

Kutterolf, S., Freundt, A., Pérez, W., Wehrmann, H., Schmincke, H.U., 2007, Late Pleistocene to Holocene temporal succession and magnitudes of highly-explosive volcanic eruptions in west-central Nicaragua. J. Volcanol. Geotherm. Res. 163: 65-83.

Lisiecki, L. E., and Raymo, M. E., 2005, A Pliocene-Pleistocene stack of 57 globally distributed benthic $\delta^{18} \mathrm{O}$ records: Paleoceanography, v. 20, no. PA1003, p. doi:10.1029/2004PA001071.

Lowe, D. J., Shane, P. A. R., Alloway, B. V., and Newnham, E. M., 2008, Fingerprints and age models for widespread New Zealand tephra marker beds erupted since 30,000 years ago: a framework for NZ-INTIMATE: Quat. Sci. Rev., v. 27, p. 95-126. 
Parson, L., Hawkins, J., and Allan, J., 1992, Lau Basin.

Mitrovica, J. X., Davis, J. L., and Shapiro, I. I., 1994, A Spectral Formalism for Computing Three-Dimensional Deformations Due to Surface Loads - I. Theory, J. Geophys. Res., v. 99, p. 7057-7073.

Peltier, W.R., 2004, Global glacial isostasy and the surface of the ice-age Earth: The ICE-5G (VM2) model and GRACE: Ann. Rev. Earth Planet. Sci., v. 32, p. 111-149.

Perez, W., and Freundt, A., 2006, The youngest highly explosive basaltic eruptions from Masaya Caldera Complex (Nicaragua): Stratigraphy and hazard assessment. In: Rose, W.I., Bluth, G., Carr, M.J., Ewert, J., Patino, L., Vallance, J. (eds) Volcanic hazards in Central America. Geol Soc Am Spec Paper 412: 189-207 doi: 10.1130/2006.2412(10)

Rangin, C., Silver, E. A., and von Breymann, M. T., 1990, Celebes and Sulu Seas.

Shipboard, S., Party, 1998, Leg 181 Preliminary Report: Southwest Pacific Gateways: ODP Prelim. Rpt., v. 181 (Online), p. Available from World Wide Web: http://wwwodp.tamu.edu/publications/prelim/181 prel/181 toc.html.

Shipboard, S., Party, 2000, Leg 190 Preliminary Report: ODP Prelim. Rpt., v. 190 (Online), p. Available from World Wide Web: http://wwwodp.tamu.edu/publications/prelim/190_prel/190toc.html.

Wu, P., and Peltier, W.R., 1982, Viscous gravitational relaxation: Geophys. J. Roy. Astron. Soc., v. 70, p. 435-486. 